\title{
Dynamical Mordell-Lang and automorphisms of blow-ups
}

\author{
John Lesieutre and Daniel Litt
}

\begin{abstract}
We show that if $\phi: X \rightarrow X$ is an automorphism of a smooth projective variety and $D \subset X$ is an irreducible divisor for which the set $\left\{d \in D: \phi^{n}(d) \in D\right.$ for some nonzero $\left.n\right\}$ is not Zariski dense in $D$, then $(X, \phi)$ admits an equivariant rational fibration to a curve. As a consequence, we show that certain blow-ups (for example, blow-ups in high codimension) do not alter the finiteness of $\operatorname{Aut}(X)$, extending results of BayraktarCantat. We also generalize results of Arnol'd on the growth of multiplicities of the intersection of a variety with the iterates of some other variety under an automorphism.

These results follow from a nonreduced analog of the dynamical Mordell-Lang conjecture. Namely, let $\phi: X \rightarrow X$ be an étale endomorphism of a smooth projective variety $X$ over a field $k$ of characteristic zero. We show that if $Y$ and $Z$ are two closed subschemes of $X$, then the set $A_{\phi}(Y, Z)=\left\{n: \phi^{n}(Y) \subseteq Z\right\}$ is the union of a finite set and finitely many residue classes; its modulus is bounded in terms of the geometry of $Y$.
\end{abstract}

\section{Introduction}

The aim of this paper is to study the group $\operatorname{Aut}(X)$ of biregular automorphisms of a projective variety $X$, a basic invariant of $X$. In particular, we analyze how $\operatorname{Aut}(X)$ changes under birational modifications of $X$.

The main result of this paper is a sort of "purity theorem" for the automorphism groupnamely, we show that its finiteness is not affected by blow-ups along smooth centers that have either large codimension or simple geometry. These considerations naturally motivate a study of the dynamics of divisors under the action of an automorphism, which we study using a generalization of the dynamical Mordell-Lang conjecture. In our view, the main contribution of this paper is the insight that this (nonreduced) dynamical Mordell-Lang conjecture places serious restrictions on the automorphism groups of blow-ups of a variety and the dynamics of those automorphisms.

The paper proceeds as follows. First, in Sections 1 and 2, we discuss the main results and illustrate them in the 2-dimensional setting. In Section 3, we formulate and prove a version of the dynamical Mordell-Lang conjecture for nonreduced schemes. Sections 4 and 5 then show how this result can be applied to study the orbits of divisors under infinite-order automorphisms. Finally,

Received 9 June 2016, accepted in final form 22 July 2017.

2010 Mathematics Subject Classification 37P55, $14 \mathrm{E} 07$.

Keywords: algebraic dynamics, dynamical Mordell-Lang conjecture, automorphisms of algebraic varieties.

This journal is (C) Foundation Compositio Mathematica 2019. This article is distributed with Open Access under the terms of the Creative Commons Attribution Non-Commercial License, which permits non-commercial reuse, distribution, and reproduction in any medium, provided that the original work is properly cited. For commercial re-use, please contact the Foundation Compositio Mathematica.

The first author was supported by NSF Research Training Group grant \#DMS-1246844. The second author was supported by an NSF Postdoctoral Fellowship. 


\section{J. Lesieutre AND D. LitT}

Sections 6 and 7 explain how these results can be applied to questions about automorphisms of varieties: we extend results of Bayraktar-Cantat on automorphisms of blow-ups [BC13] and show how some results of Arnol'd [Arn93] can be generalized and understood as an instance of a nonreduced version of the dynamical Mordell-Lang conjecture.

We first survey the main results. Throughout, we work over an arbitrary algebraically closed field $k$ of characteristic zero. A variety is an integral scheme of finite type over $k$, not assumed to be proper. Many of our results hold without any properness assumptions, but some results requiring global geometric tools require projectivity.

The usual form of the dynamical Mordell-Lang conjecture is the following.

Conjecture 1.1 (Dynamical Mordell-Lang conjecture). Suppose that $\phi: X \rightarrow X$ is an endomorphism of a quasiprojective variety $X$ over $k$, with $V \subset X$ a subvariety and $p \in X$ a point. Then the set

$$
A_{\phi}(p, V)=\left\{n: \phi^{n}(p) \in V\right\}
$$

is a union of a finite set and a finite number of arithmetic progressions.

When $\phi$ is étale, the conjecture is a theorem of Bell-Ghioca-Tucker [BGT10]. Our first result extends this result in the étale case to the setting in which $p$ and $V$ are replaced by arbitrary closed subschemes of $X$.

Theorem 1.2. Suppose that $X$ is a smooth variety over $k$ and that $\phi: X \rightarrow X$ is an étale endomorphism of $X$. Let $Y$ and $Z$ be closed subschemes of $X$. Then the set $A_{\phi}(Y, Z)=\left\{n: \phi^{n}(Y) \subseteq Z\right\}$ is a union of a finite set and a finite number of arithmetic progressions.

Here $\phi^{n}(Y)$ denotes the scheme-theoretic image of $Y$ under $\phi^{n}$, and the containment $\phi^{n}(Y) \subseteq Z$ is a scheme-theoretic inclusion, not a set-theoretic inclusion.

This theorem was proved by Bell-Lagarias for affine $X$ [BL15]. For our geometric applications, it will be important to have some control over how the lengths of the arithmetic progressions appearing in $A_{\phi}(Y, Z)$ depend on $Y$ and $Z$.

Theorem 1.3. Let $X, \phi$, and $Z$ be as in Theorem 1.2. Suppose that $\left\{Y_{1}^{\circ}, \ldots, Y_{r}^{\circ}\right\}$ is a finite set of closed subschemes of $X$, defined by ideal sheaves $\mathscr{I}_{Y_{i}^{\circ}}$, and that $\left\{Y_{j}\right\}$ is an infinite collection of subschemes defined by ideal sheaves of the form

$$
\sum_{i=1}^{r} \mathscr{I}_{Y_{i}^{\circ}}^{n_{i}},
$$

with $n_{i} \geqslant 0$. Let $\Xi$ be the set of all associated points of the $Y_{j}$.

(i) Suppose that $x$ is a point of $X$ with $x \in \bar{\xi}$ for only finitely many $\xi \in \Xi$. Then there exists a constant $N=N\left(\left\{Y_{j}\right\}, Z, x\right)$ (in particular, independent of $j$ ) such that the set of $n$ for which $\phi^{n}\left(Y_{j}\right) \subseteq Z$ in $\operatorname{Spec}\left(\mathscr{O}_{X, x}\right)$ can be expressed as the union of arithmetic progressions of length bounded by $N$ and a finite set.

(ii) Suppose that the set of associated points $\Xi$ is finite. Then there exists a constant $N=$ $N\left(\left\{Y_{j}\right\}, Z\right)$ such that the lengths of the arithmetic progressions appearing in $A_{\phi}\left(Y_{j}, Z\right)$ are bounded by $N$ (independently of $j$ ).

Remark. Note that the point $x$ in part (i) above need not be a closed point.

Remark. The important feature of ideals of the form $\sum_{i=1}^{r} \mathscr{I}_{Y_{i}^{\circ}}^{n_{i}}$ is that if the ideals $\mathscr{I}_{Y_{i}^{\circ}}$ are defined over a given finitely generated extension of $\mathbb{Z}$, then so too are all of the $\left\{Y_{j}\right\}$. In fact, the 


\section{Dynamical Mordell-Lang AND AUtomorphisms of BlOW-UPS}

claim of the theorem holds more generally if the $Y_{j}$ are constructed from a finite set of ideals using any combination of operations which do not change the ring of definition. This includes not only the formation of sums and products, but also taking primary components (and in particular taking symbolic powers).

Remark. The behavior of the periods $A_{\phi}\left(Y_{j}, Z\right)$ can be quite subtle when the set $\left\{Y_{j}\right\}$ is infinite, and some hypotheses on associated points are necessary for part (ii) to hold. In Example 2.6 below, we exhibit an automorphism $\phi: X \rightarrow X$, a subscheme $Z \subset X$, and an infinite set of closed subschemes $Y_{j}$ which all have the same support, but for which the periods of $A_{\phi}\left(Y_{j}, Z\right)$ grow without bound due to the presence of embedded points.

We are primarily motivated by geometric applications of Theorem 1.3 , which does not have an analog in the setting where $Y$ and $Z$ are reduced. The uniform bound on the period makes it possible to control the multiplicities of the intersection of a subvariety $V$ with the iterates $\phi^{n}(V)$ under an automorphism.

If $x$ is the generic point of a codimension 2 variety of $X$, then the hypotheses of point (i) hold automatically: an associated point of $Y_{j}$ whose closure contains $x$ must be either $x$ itself, or the generic point of a divisor containing $x$. But only finitely many divisors appear among the support of the $Y_{j}$. However, in general, the set $\Xi$ need not be finite; see Example 2.7 below. Nevertheless, this hypothesis is satisfied in a number of natural geometric settings, discussed in Theorem 3.9.

The first geometric application of these results is the following.

Theorem 1.4. Let $X$ be a smooth variety over $k$ and $\phi: X \rightarrow X$ an automorphism. Suppose that $D \subset X$ is an irreducible divisor containing an irreducible codimension 2 closed subset $V \subset X$ with $\phi(V)=V$. If $D$ is not $\phi$-periodic, then there exists a projective birational morphism $\pi: Y \rightarrow X$ such that

(i) $Y$ is smooth;

(ii) $\pi$ is an isomorphism away from $V$;

(iii) some iterate of $\phi$ lifts to an automorphism $\psi: Y \rightarrow Y$;

(iv) $\pi\left(\psi^{m}(\tilde{D}) \cap \psi^{n}(\tilde{D})\right)$ does not contain $V$ for any $m \neq n$.

Here $\tilde{D}$ is the strict transform of $D$ on $Y$. In part (iii) above, the statement that $\phi: X \rightarrow X$ lifts through $\pi$ to an automorphism $\psi: Y \rightarrow Y$ (equivalently, $\psi$ descends to $\phi$ ) means that there is a commutative diagram

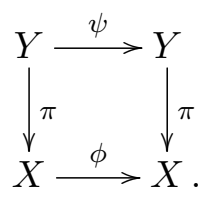

As $\pi$ is an isomorphism over a Zariski-open (hence Zariski-dense) subset of $X$, there is at most one lift $\psi$ of any automorphism $\phi$ of $X$ (equivalently, at most one descent of $\psi$ to an automorphism $\phi$ ).

If the intersection of $D$ with the union of the divisors $\phi^{n}(D)$ is a proper Zariski-closed subsets of $D$, repeated applications of this local separation result make it possible to blow up $X$ several times and simultaneously make the strict transforms of all of the divisors $\phi^{n}(D)$ disjoint. Global geometric considerations then yield the following result. 


\section{J. LeSiEUTRE AND D. LitT}

Theorem 1.5. Let $X$ be a smooth projective variety and $\phi: X \rightarrow X$ an automorphism. Suppose that $D \subset X$ is an irreducible divisor for which the set

$$
V(D)=\left\{d \in D: \phi^{n}(d) \in D \text { for some nonzero } n\right\}=\bigcup_{n \neq 0} D \cap \phi^{n}(D)
$$

is a proper, Zariski-closed subset of $D$. Then there exists a projective birational morphism $\pi: Y \rightarrow X$ such that

(i) $Y$ is smooth;

(ii) some iterate of $\phi$ lifts to an automorphism $\psi: Y \rightarrow Y$;

(iii) the divisors $\psi^{n}(\tilde{D})$ are pairwise disjoint;

(iv) there exist a curve $C$, a morphism $f: Y \rightarrow C$, and an automorphism $\tau: C \rightarrow C$ such that $f \circ \psi=\tau \circ f$.

Moreover, the normal bundle of $D$ satisfies $H^{0}\left(D, N_{D / X}\right)>0$ and $D$ moves in a positivedimensional family on $X$.

Theorem 1.5 can then be used to address concrete questions about automorphisms of varieties constructed using sequences of blow-ups.

Theorem 1.6. Suppose that $X$ is a smooth projective variety of dimension $n$ and that $Y$ is a variety constructed by a sequence of smooth blow-ups of $X$. If each blow-up $\pi_{i}: X_{i+1} \rightarrow X_{i}$ in the sequence, with exceptional divisor $E$ and center of dimension $r=\operatorname{dim} \pi_{i}(E)$, satisfies either

(i) $2 r+3 \leqslant n$, or

(ii) $r+3 \leqslant n$ and $\operatorname{Nef}(E)$ is a polyhedral cone,

then there exists an integer $N \geqslant 1$ such that for any automorphism $\phi$ of $Y$, the iterate $\phi^{N}$ descends to an automorphism of $X$.

This was proved for blow-ups of type (i) by Bayraktar and Cantat under the additional hypothesis $\rho(X)=1$ [BC13]. Here we present a different proof which does not require this hypothesis. Although the proof in case (i) is independent of our main results, the handling of case (ii) relies on the results of the preceding sections. Observe that a blow-up at a center of Picard rank 1 automatically satisfies statement (ii). The fact that the number $N$ in Theorem 1.6 does not depend on $\phi$ is useful in applications, particularly Corollary 1.9.

Observe that part (ii) of Theorem 1.6 implies that blow-ups of fourfolds along points and curves cannot obtain new infinite-order automorphisms.

Corollary 1.7. Suppose that $X$ is a smooth projective variety of dimension 4 and that $Y$ is a variety constructed by a sequence of blow-ups of points and smooth curves in $X$. Then there exists an integer $N \geqslant 1$ such that for any automorphism $\phi$ of $Y$, the iterate $\phi^{N}$ descends to an automorphism of $X$.

The conclusion of Theorem 1.6 is false when $r=n-2$, for every value of $n$ (see Example 2.5 below), but the situation for $\lfloor(n-1) / 2\rfloor \leqslant r \leqslant n-3$ remains unclear. It seems natural to ask the following.

Question 1.8. Do there exist a projective variety $X$ with $\operatorname{Aut}(X)$ a finite group and a sequence of smooth blow-ups $\pi: Y \rightarrow X$ along smooth centers of codimension at least 3 such that $\operatorname{Aut}(Y)$ is infinite? 


\section{Dynamical Mordell-LANG AND AUtomorphisms OF BLOW-UPS}

Corollary 1.9 below shows that the answer is negative for $\operatorname{dim}(X) \leqslant 4$.

Corollary 1.9. Let $X$ and $Y$ be as in Theorem 1.6. Then if $\operatorname{Aut}(X)$ has finite component group, the same is true for $\operatorname{Aut}(Y)$.

Truong has also obtained a variety of results in this direction, under some additional hypotheses on the nef cone of $Y$ (for example, [Tru17, Lemma 6]). Arguments of a similar flavor appeared in the first author's paper [Les15], where the 3-dimensional case of Theorem 1.4 was proved by different methods. The results on automorphisms in [Les15] are specific to positive entropy automorphisms in dimension 3; much stronger conclusions can be obtained in that setting.

\section{Some examples}

Before giving the proof of Theorem 1.3, we give an indication of its geometric content in the case that $Y$ is a nonreduced subscheme. For simplicity, suppose that $X$ is a smooth algebraic surface and that $Y$ and $Z$ are two smooth curves on $X$ which intersect at $V$, a fixed point of an automorphism $\phi: X \rightarrow X$.

Let $Y^{(1)}$ be the first-order germ of $Y$ at $V$, a closed subscheme given by an embedding Spec $\mathbb{C}[\epsilon] /\left(\epsilon^{2}\right) \rightarrow X$. We have $\phi^{n}\left(Y^{(1)}\right) \subseteq Z$ exactly when $\phi^{n}(Y)$ is tangent to $Z$ at the fixed point $V$.

Theorem 1.2 applied to the scheme $Y^{(1)}$ then asserts that

$$
\begin{aligned}
A_{\phi}\left(Y^{(1)}, Z\right) & =\left\{n: \phi^{n}\left(Y^{(1)}\right) \subseteq Z\right\} \\
& =\left\{n: \phi^{n}(Y) \text { is tangent to } Z \text { at } V\right\}
\end{aligned}
$$

is a union of a finite set and finitely many arithmetic progressions.

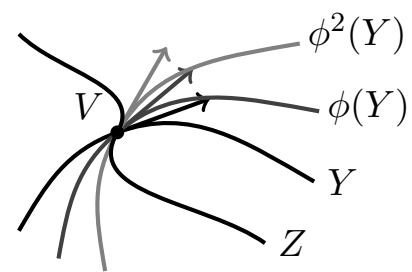

Figure 1 . The curve $\phi^{n}(Y)$ is tangent to $Z$ at $V$ when $\phi^{n}\left(Y^{(1)}\right) \subset Z$.

More generally, take $Y_{1}^{\circ}=C$ and $Y_{2}^{\circ}=V$, and consider the collection of schemes $Y^{(j)}$ defined by the ideal sheaves

$$
\mathscr{I}_{Y_{1}^{\circ}}+\mathscr{I}_{Y_{2}^{\circ}}^{j+1} \text {. }
$$

The scheme $Y^{(1)}$ is precisely the one considered above, while $Y^{(j)}$ corresponds to a $j$ th-order germ of the curve $C$ at $V$. The collection of closed subschemes $Y^{(j)}$ is infinite, but it satisfies the hypotheses of Theorem 1.3(ii).

Applying the theorem to the higher-order germs $Y^{(j)}$ of $Y$ at $V$, we find that

$$
\begin{aligned}
A_{\phi}\left(Y^{(j)}, Z\right) & =\left\{n: \phi^{n}\left(Y^{(j)}\right) \subseteq Z\right\} \\
& =\left\{n: \phi^{n}(Y) \text { is tangent to } Z \text { at } V \text { to order } \geqslant j\right\}
\end{aligned}
$$

is the union of a finite set and finitely many arithmetic progressions, and there exists a bound $N$ on the periods of the arithmetic progressions occurring in these sets which is independent of $j$. 


\section{J. LESIEUTRE AND D. LitT}

This implies, in particular, that there is a uniform bound on the order of tangency between $C$ and $\phi^{n}(C)$ for nonzero $n$ : if $C$ is ever tangent to some $\phi^{n}(C)$ to order at least some $j$, then such a tangency must occur within the first $N$ iterates or within the finite set. More general statements in this direction appear as Lemmas 4.4 and 4.5.

We now turn our attention to Theorem 1.4 in this setting: the theorem asserts that it is possible to blow up above the point $V$ a finite number of times and reach a model $X^{\prime}$ such that $\phi$ lifts to an automorphism of $X^{\prime}$ and the curves $\phi^{n}(Y)$ simultaneously become disjoint above $V$. These two objectives are in tension: if we do not blow up enough, the curves $\phi^{n}(C)$ will not be disjoint; on the other hand, if we blow up too aggressively, the automorphism $\phi$ will not lift. The essential obstacle to constructing the requisite blow-ups is that the order of tangency between $Y$ and the $\phi^{n}(Y)$ might not be bounded in $n$.

To overcome this difficulty, we must show that for any $k$, the curve $Y$ is tangent to $\phi^{n}(Y)$ to order at least $k$ for all $n$ in some arithmetic progression, and that the length of this progression is independent of $k$. But this is precisely the content of Theorem 1.3, applied to the schemes $Y^{(j)}$ above.

The next two examples give automorphisms to which Theorem 1.5 is applicable, and in which conclusions of the theorem can easily be seen.

Example 2.1. Let $X=\mathbb{P}^{2}$, and let $\phi: \mathbb{P}^{2} \rightarrow \mathbb{P}^{2}$ be an automorphism of infinite order. Take $D$ to be a general line through a fixed point $x$. Then $D$ has infinite order under $\phi$, but $V_{D}=$ $\bigcup_{n \neq 0} D \cap \phi^{n}(D)=\{x\}$ is Zariski closed.

This is consistent with the last part of Theorem 1.5: Take $\pi: Y \rightarrow X$ to be the blow-up at $x$. Then $\phi$ lifts to an automorphism $\psi$ of $Y$, and the divisors $\psi^{n}(\tilde{D})$ are pairwise disjoint. The pencil of lines through $x$ induces a map $f: Y \rightarrow \mathbb{P}^{1}$, and there is an automorphism of $\tau: \mathbb{P}^{1} \rightarrow \mathbb{P}^{1}$ with $f \circ \psi=\tau \circ f$. Note that $H^{0}\left(D, N_{D / X}\right)=H^{0}\left(D, \mathscr{O}_{D}(1)\right)>0$, as required.

The next example shows that although $N_{D / X}$ must be effective, in general, it need not have any stronger sort of positivity (for example, nefness or movability).

Example 2.2. Let $\psi: \mathbb{P}^{3} \rightarrow \mathbb{P}^{3}$ be an infinite-order automorphism, with fixed points $p_{i}$. Let $\pi: X \rightarrow \mathbb{P}^{3}$ be the blow-up of $\mathbb{P}^{3}$ at the points $p_{1}$ and $p_{2}$, with exceptional divisors $E_{1}$ and $E_{2}$. Then $\psi$ induces an automorphism $\phi: X \rightarrow X$. Let $D$ be the strict transform on $X$ of a general plane in $\mathbb{P}^{3}$ passing through the points $p_{1}$ and $p_{2}$, and let $L$ be the strict transform of the line between these two points. Then $V_{D}=L$ is not Zariski dense.

In this case, $\left.N_{D / X} \cong\left(\pi^{*} \mathscr{O}_{\mathbb{P}^{3}}(1) \otimes \mathscr{O}_{X}\left(-E_{1}-E_{2}\right)\right)\right|_{D}$ is not nef, since it has negative intersection with $L$. This shows that the conclusion of Theorem 1.5 that $N_{D / X}$ is effective cannot be strengthened to include the conclusion that it is nef or even movable.

It follows from Theorem 1.5 that if $\phi: X \rightarrow X$ is an automorphism of a projective variety and $D \subset X$ is any irreducible divisor which is not the fiber of any $\phi^{k}$-equivariant fibration, then $\bigcup_{n \neq 0} D \cap \phi^{n}(D)$ is a Zariski-dense subset of $D$. However, this set is typically not analytically dense.

Example 2.3. Suppose that $\phi: X \rightarrow X$ is an automorphism of a surface. Let $x \in X$ be an attracting fixed point of $\phi$ with basin of attraction the (analytically) open set $W^{s}(x) \subset X$, and let $y \in X$ be an attracting fixed point of $\phi^{-1}$ with basin of attraction $W^{u}(y) \subset X$. Suppose too that $W^{s}(x) \cap W^{u}(y)$ is nonempty.

Let $C \subset X$ be a curve meeting $W^{s}(x) \cap W^{u}(y)$ but not passing through the points $x$ or $y$. Choose a point $z \in C \cap W^{s}(x) \cap W^{u}(y)$, and let $B \subset X$ be a closed ball around $z$ which is contained 
in $W^{s}(x) \cap W^{u}(y)$. Fix a number $N$ and compact sets $K_{x}$ containing $x$ and $K_{y}$ containing $y$ with $K_{x} \cap C$ and $K_{y} \cap C$ empty and such that if $n>N$, we have $\phi^{n}(B) \subset K_{x}$ and $\phi^{-n}(B) \subset K_{y}$.

Let $B^{0}$ be the interior of $B$, and consider the open subset $A=B^{0} \cap C \subset C$. If $|n|>N$ and $a$ is any point of $A$, we have either $\phi^{n}(a) \in K_{x}$ or $\phi^{n}(a) \in K_{y}$, so that in particular $\phi^{n}(a)$ does not lie on $C$. Then $A \backslash\left(\bigcup_{n=-N}^{N} \phi^{n}(C) \cap C\right)$ is an (analytically) open subset of $C$ which is disjoint from $\bigcup_{n \neq 0} C \cap \phi^{n}(C)$, but in general $C$ will not be a fiber of an invariant rational fibration.

It bears noting that Theorem 1.2 contains the classical Skolem-Mahler-Lech theorem on arithmetic progressions as a special case. As a result, it comes as no surprise that the proof relies on methods of $p$-adic analysis.

Example 2.4 ([BGT10]). Consider $X=\mathbb{P}^{2}$ with coordinates $\left[W_{0}, W_{1}, W_{2}\right]$, and let $M: \mathbb{P}^{2} \rightarrow \mathbb{P}^{2}$ be the linear automorphism given by the matrix

$$
M=\left[\begin{array}{ccc}
0 & 1 & 0 \\
0 & 0 & 1 \\
c_{3} & c_{2} & c_{1}
\end{array}\right] .
$$

Let $Y$ be the point $\left[x_{0}, x_{1}, x_{2}\right]$, and let $Z$ be the hyperplane $W_{0}=0$. Then $\phi^{n}(Y)$ is the point $\left[x_{n}, x_{n+1}, x_{n+2}\right]$, where $x_{n}$ is defined by the linear recurrence sequence $x_{n}=c_{1} x_{n-1}+c_{2} x_{n-2}+$ $c_{3} x_{n-3}$. Then $\phi^{n}(Y) \subseteq Z$ exactly when $x_{n}=0$, and the theorem asserts that the set of such $n$ is the union of a finite set and an arithmetic progression. This is the classical Skolem-Mahler-Lech theorem.

Recall that the Skolem-Mahler-Lech theorem is false over a field $k$ of positive characteristic, and so our main results cannot be extended to that setting.

Automorphisms of blow-ups of $\mathbb{P}^{2}$ at configurations of 9 or more points are an important source of examples. However, analogous examples in higher-dimensional settings are little understood. The result of Theorem 1.7 strengthens dimensional constraints on such examples due to Bayraktar and Cantat.

Example 2.5. Let $C_{1}$ and $C_{2}$ be two general cubics in $\mathbb{P}^{2}$, and let $X$ be the blow-up of $\mathbb{P}^{2}$ at the 9 points of $C_{1} \cap C_{2}$, with exceptional divisors $E_{0}, \ldots, E_{8}$. The pencil of cubics induces an elliptic fibration $\pi: X \rightarrow \mathbb{P}^{1}$, and the divisors $E_{i}$ determine sections of $\pi$. Take the image of $E_{0}$ to give a base point on each fiber. Fiberwise addition $x \mapsto E_{i}+x$ using the group law of the fibers gives infinite-order automorphisms $\tau_{i}: X \rightarrow X(1 \leqslant i \leqslant 8)$.

Taking products $\mathbb{P}^{2} \times \mathbb{P}^{n-2}$, we obtain examples in any dimension $n \geqslant 2$ of automorphisms of blow-ups along codimension 2 subsets which admit automorphisms of infinite order.

The next two examples illustrate some of the subtleties of dynamical Mordell-Lang statements in the setting of nonreduced schemes; the existence of embedded points creates new complications. The first example gives an automorphism $\phi: X \rightarrow X$ and an infinite collection of closed subschemes $Y_{j} \subset X$ such that the periods of the arithmetic progressions in the sets $A_{\phi}\left(Y_{j}, Z\right)$ are not uniformly bounded. This necessitates that we consider only subschemes $Y_{j}$ of a restricted form, as in Theorem 1.3.

Example 2.6. Let $g: S \rightarrow S$ be an automorphism of a projective variety for which there exist periodic points of all periods $k \geqslant 0$. For example, we may take $E$ to be an elliptic curve and $S=E \times E$. Then the map $g: S \rightarrow S$ given by the matrix $\left(\begin{array}{ll}1 & 1 \\ 0 & 1\end{array}\right)$ has periodic points of all possible 


\section{J. LESIEUTRE AND D. LitT}

periods: if $x_{k}$ is an $k$-torsion point on $E$, then $\phi^{k}\left(0, x_{k}\right)=\left(k x_{k}, x_{k}\right)=\left(0, x_{k}\right)$, and so this point has period $k$.

Now, let $X=S \times S \times \mathbb{P}^{1}$ and consider the automorphism $\phi=\operatorname{id}_{S} \times \phi \times \operatorname{id}_{\mathbb{P}^{1}}: X \rightarrow X$. Let $Z$ be the subscheme defined by $\mathscr{I}_{S \times S \times 0} \cdot \mathscr{I}_{\Delta \times 0}^{2}$, giving $S \times S \times 0$ with nilpotents in the structure sheaf along the diagonal $\Delta \subset S \times S$. Then consider the subschemes $Y_{k}$ defined by $\mathscr{I}_{S \times S \times 0} \cdot \mathscr{I}_{\left(y_{k}, y_{k}, 0\right)}^{2}$, where $y_{k}$ is a $g$-periodic point of period $k$. The associated points of $Y_{k}$ are the generic point of $S \times S \times 0$ and embedded point $\left(y_{k}, y_{k}, 0\right)$.

Observe that $\mathscr{I}_{\phi^{n}\left(Y_{k}\right)}=\mathscr{I}_{S \times S \times 0} \cdot \mathscr{I}_{\phi\left(y_{k}, y_{k}, 0\right)}^{2}=\mathscr{I}_{S \times S \times 0} \cdot \mathscr{I}_{\left(x_{k}, \phi^{n}\left(x_{k}\right), 0\right)}^{2}$ since $\phi^{n}\left(y_{k}, y_{k}, 0\right)=$ $\left(y_{k}, \phi^{n}\left(y_{k}\right), 0\right)$. Then $\phi^{n}\left(Y_{k}\right)$ is contained in $Z$ if and only if the embedded point $\left(y_{k}, \phi^{n}\left(y_{k}\right), 0\right)$ is contained in $Z$, which is the case exactly when $\left(y_{k}, \phi^{n}\left(y_{k}\right), 0\right)$ lies on the diagonal $\Delta \times 0 \subset X$, so that $\phi^{n}\left(y_{k}\right)=y_{k}$. In particular, we have $\phi^{n}\left(Y_{k}\right) \subset Z$ only when $n$ is a multiple of $k$.

Example 2.7. The hypothesis that the set $\Xi$ is finite in Theorem 1.3 need not hold in general for subschemes defined by ideals of the form $\sum_{i=1}^{r} \mathscr{I}_{Y_{i}^{\circ}}^{n_{i}}$. This is illustrated by the following example of Singh and Swanson [SS04, Theorem 4.6]. For this reason, it may sometimes be preferable to work with primary components of such sums instead, though we will not find this necessary in our applications.

Let $k$ be an algebraically closed field, and let $X$ be the hypersurface in $\mathbb{A}^{4}=\operatorname{Spec} k[w, x, y, z]$ defined by $w y^{2}+x y z+w z^{2}=0$. Consider the subschemes defined by the ideal sheaves $\mathscr{I}_{Y_{1}^{\circ}}=(w)$ and $\mathscr{I}_{Y_{2}^{\circ}}=(x)$. It is shown in [SS04] that for any $n$, the associated points of the closed subscheme defined by $\mathscr{I}_{Y_{1}^{\circ}}^{n}+\mathscr{I}_{Y_{2}^{\circ}}^{n}$ correspond to the primes

$$
(y, z),(x, y, z),(w, x, y, z),\left(y, z, x-w \xi-w \xi^{-1}\right),
$$

where $\xi$ ranges among the $n$th roots of unity. As $n$ ranges over all positive integers, the set of associated points is thus infinite. Here we have two surfaces contained in a threefold, intersecting along a curve. The thickenings of the surfaces defined by powers of the ideals have intersections with various embedded points along the curve.

\section{The dynamical Mordell-Lang theorem in the nonreduced case}

In this section, we prove Theorem 1.3. The ideas are more or less the same as in the work of Bell-Lagarias [BL15] and Bell-Ghioca-Tucker [BGT15], with two minor complications. First, by reducing to the case of complete local rings of an arithmetic scheme, we are able to treat the general case, rather than only the affine case. Second, Theorem 1.3 (and our applications of it) requires uniform bounds on periods for an infinite collection of subschemes, necessitating careful consideration of the associated points of those subschemes.

Definition 3.1. A subset $A \subset \mathbb{Z}$ is said to be a one-sided semilinear set if there is a decomposition

$$
A=F \cup\left(\bigcup_{i=1}^{m} P_{i}\right),
$$

where $F$ is a finite set and each $P_{i}=a_{i}+b_{i} \mathbb{Z}_{\geqslant 0}$ is a one-sided arithmetic progression. Similarly, $A \subset \mathbb{Z}$ is called a (two-sided) semilinear set if

$$
A=F \cup\left(\bigcup_{i} P_{i}\right),
$$

where $F$ is a finite set and each $P_{i}=a_{i}+b_{i} \mathbb{Z}$ is a two-sided arithmetic progression. 


\section{Dynamical Mordell-Lang AND AUtomorphisms of BlOW-UPS}

We say that a one-sided or two-sided semilinear set $A$ is $N$-periodic if there exists a decomposition for which the period $b_{i}$ of each $P_{i}$ divides $N$.

Lemma 3.2. Suppose that $\left\{A_{i}\right\}$ is a (possibly infinite) collection of $N$-periodic semilinear sets. Then $\bigcap_{i} A_{i}$ is an $N$-periodic semilinear set.

Proof. Observe that if $A$ is an $N$-periodic semilinear set, then it can be written as the union of a finite set and a finite set of arithmetic progressions of length exactly $N$ : indeed, if $k$ divides $N$, an arithmetic progression of length $k$ can be subdivided into $N / k$ progressions of length $N$. For each $A_{i}$, write

$$
A_{i}=F_{i} \cup\left(\bigcup_{j} P_{i, j}\right),
$$

where each $P_{i, j}$ is an arithmetic progression with period $N$. Then

$$
\bigcap_{i} A_{i}=\left(\bigcup_{0 \leqslant m \leqslant N}(m+N \mathbb{Z})\right) \cap \bigcap_{i} A_{i}=\bigcup_{0 \leqslant m \leqslant N}\left((m+N \mathbb{Z}) \cap \bigcap_{i} A_{i}\right) .
$$

Note that $(m+N \mathbb{Z}) \cap A_{i}=m+N \mathbb{Z}$ if $m+N \mathbb{Z}$ is among the arithmetic progressions $P_{i, n}$, while $(m+N \mathbb{Z}) \cap A_{i}=(m+N \mathbb{Z}) \cap F_{i}$ is a finite set otherwise. It follows that $(m+N \mathbb{Z}) \cap A_{i}=m+N \mathbb{Z}$ is either $m+N \mathbb{Z}$ or finite, and so

$$
\bigcup_{0 \leqslant m \leqslant N}\left((m+N \mathbb{Z}) \cap \bigcap_{i} A_{i}\right)
$$

is a union of finite sets and arithmetic progressions of length $N$.

We now prove a local version of our dynamical Mordell-Lang theorem.

Lemma 3.3 (Local dynamical Mordell-Lang theorem). Let $K$ be a finite extension of $\mathbb{Q}_{p}$ with valuation ring $R$, uniformizer $\pi$, and residue field $\kappa$, and let $f \in R\left\langle x_{1}, \ldots, x_{n}\right\rangle^{n}$ be an $n$-tuple of convergent power series (that is, the p-adic absolute values of the coefficients tend to zero) inducing a topological isomorphism

$$
R\left[\left[x_{1}, \ldots, x_{n}\right]\right] \rightarrow R\left[\left[x_{1}, \ldots, x_{n}\right]\right] .
$$

Suppose that $f$ is affine linear $\bmod \pi$.

Then for any two closed formal subschemes $Y$ and $Z$ of $\operatorname{Spf}\left(R\left[\left[x_{1}, \ldots, x_{n}\right]\right]\right)$, the set of $m \in \mathbb{Z}$ such that $f^{m}(Y) \subseteq Z$ is a two-sided semilinear set. Furthermore, the semilinear set is $N$-periodic for some $N$ depending only on $\#|k|$ and $|\pi|_{p}$ (and not on $Y, Z$, or $f$ ).

Proof. By the affine-linearity assumption,

$$
f(\mathbf{x})=A \mathbf{x}+\mathbf{b} \bmod \pi
$$

for some $A \in \mathrm{GL}_{n}(\kappa)$ and $\mathbf{b} \in \kappa^{n}$. As the group of affine-linear transformations of $\kappa^{n}$ is finite, there exists some $N$ such that

$$
f^{N}=\mathbf{x} \bmod \pi \text {. }
$$

(Observe that this $N$ depends only on $\#|\kappa|$.) After possibly increasing $N$ (say, replacing it with $p^{M} N$ for some $M \gg 0$ depending only on $|\pi|_{p}$ ), we may assume that

$$
f^{N}=\mathbf{x} \bmod \pi^{a}
$$

for any fixed $a$. See for example [Poo14, Remark 4]. 


\section{J. Lesieutre AND D. LitT}

We may now apply the main result of [Poo14] to obtain an element

$$
g \in R\left\langle x_{1}, \ldots, x_{n}, m\right\rangle
$$

such that

$$
g(\mathbf{x}, r)=f^{N r}(\mathbf{x})
$$

for any $r \in \mathbb{Z}$. Given any $\gamma \in R$, the element $g$ induces a map

$$
g(-, \gamma): R\left[\left[x_{1}, \ldots, x_{n}\right]\right] \stackrel{g(\mathbf{x}, \gamma)}{\longrightarrow} R\left[\left[x_{1}, \ldots, x_{n}\right]\right] .
$$

Fixing any element $h \in \mathscr{I}_{Z}$, the induced function

$$
\begin{aligned}
g(h,-): \mathbb{Z}_{p} & \rightarrow R\left[\left[x_{1}, \ldots, x_{n}\right]\right] \\
\gamma & \mapsto g(h, \gamma)
\end{aligned}
$$

is $p$-adic analytic. Let $q: R\left[\left[x_{1}, \ldots, x_{n}\right]\right] \rightarrow \mathscr{O}_{Y}$ be the natural quotient map.

Let $m=N r+s$. Now, $f^{m}(Y) \subseteq Z$ if and only if every function vanishing on $Z$ vanishes on $f^{m}(Y)$, that is, if and only if $q \circ f^{s} \circ g(h, r)=0$ for all $h \in \mathscr{I}_{Z}$. But this last is a $p$-adic analytic function of $r$; hence it either has finitely many zeros or is identically zero. It follows that the set of zeros of $q \circ f^{s} \circ g(h,-)$ is an $N$-periodic semilinear set for every $h$ in $\mathscr{I}_{Z}$. By Lemma 3.2, the intersection of these sets for all $h \in \mathscr{I}_{Z}$ is an $N$-periodic semilinear set as well. This proves the theorem.

Remark. When we say that a function $f$ whose target is an $R$-module $M$ of infinite rank (for example, the function $g(h,-)$ above) is $p$-adic analytic, we mean that for every continuous $R$-linear form $\ell: M \rightarrow R$, the composition $\ell \circ f$ is $p$-adic analytic.

Before proceeding to the global situations (Theorems 1.2 and 1.3), we need two lemmas which will allow us to reduce to Lemma 3.3.

Lemma 3.4 ([BGT10, Proposition 2.2]). Let $K$ be a finite extension of $\mathbb{Q}_{p}$ with valuation ring $R$, uniformizer $\pi$, and residue field $\kappa$. Let $X$ be a smooth, finite-type $R$-scheme with geometrically connected fibers.

Let $\phi: X \rightarrow X$ be an étale $R$-map, and let $x \in X(\kappa)$ be a point with $\phi(x)=x$. Then there exists an isomorphism

with $n=\operatorname{dim}(X)$ such that

$$
\widehat{\mathscr{O}_{X, x}} \simeq R\left[\left[x_{1}, \ldots, x_{n}\right]\right]
$$

(i) the automorphism of $R\left[\left[x_{1}, \ldots, x_{n}\right]\right]$ induced by $\phi$ is given by some $f \in R\left\langle x_{1}, \ldots, x_{n}\right\rangle^{n}$, and

(ii) the function $f$ is affine linear $\bmod \pi$.

Proof. The proof is exactly the same as that of [BGT10, Proposition 2.2], replacing $p$ with $\pi$.

Lemma 3.5. Let $R$ be a discrete valuation ring and $X / R$ a separated, finite-type $R$-scheme. Let $Y$ and $Z$ be closed subschemes of $X$. Suppose that $Y$ has exactly one associated point (in particular, $Y$ is irreducible). Let $y \in Y$ be any closed point with ideal sheaf $\mathfrak{m}_{y}$; let $y_{n} \subset Y$ be the closed subscheme associated with $\mathfrak{m}_{y}^{n}$. Then $Y \subset Z$ if and only if $y_{n} \subset Z$ for all $y$ and $n$.

Proof. The hypothesis is that $\mathscr{O}_{X} \rightarrow \widehat{\mathscr{O}_{Y, y}} / \mathfrak{m}_{y}^{n}$ has $\mathscr{I}_{Z}$ in its kernel for all $n$, in other words, that the composition

$$
\mathscr{I}_{Z} \rightarrow \mathscr{O}_{X} \rightarrow \widehat{\mathscr{O}_{Y, y}}
$$




\section{Dynamical Mordell-Lang AND AUtomorphisms of BLOW-UpS}

is zero. We wish to show that $\mathscr{I}_{Z}$ is in the kernel of the quotient map $\mathscr{O}_{X} \rightarrow \mathscr{O}_{Y}$. But consider the diagram

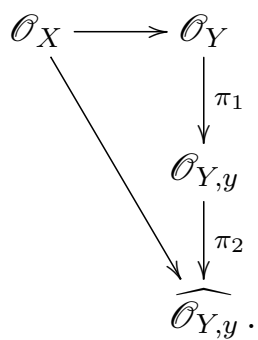

We observe that $\pi_{1}$ is injective as $Y$ has one associated point, and $\pi_{2}$ is injective by Krull's intersection theorem. Thus,

$$
\operatorname{ker}\left(\mathscr{O}_{X} \rightarrow \widehat{\mathscr{O}_{Y, y}}\right)=\operatorname{ker}\left(\mathscr{O}_{X} \rightarrow \mathscr{O}_{Y}\right)
$$

completing the proof.

Corollary 3.6. Let $R$ be a discrete valuation ring and $X / R$ a separated, finite-type $R$-scheme. Let $Y$ and $Z$ be closed subschemes of $X$. Let $y_{i} \subset Y$ be a collection of closed points with ideal sheaf $\mathfrak{m}_{y_{i}}$, such that the closure of each associated point of $Y$ contains some $y_{i}$. Then $Y \subset Z$ if and only if $Y \cap \operatorname{Spf}\left(\widehat{\mathscr{O}_{Y, y_{i}}}\right) \subset Z \cap \operatorname{Spf}\left(\widehat{\mathscr{O}_{Y, y_{i}}}\right)$ for all $i$.

Proof. The case that $Y$ has one associated point is exactly Lemma 3.5. Without loss of generality, we may suppose that $y_{1} \subset Y$ has no other associated points in its closure. Let $\mathscr{I}$ be the ideal sheaf in $\mathscr{O}_{Y}$ consisting of functions whose support is contained in $\overline{y_{1}}$. Then $Y$ is contained in $Z$ if and only if both $\operatorname{Spec}_{X}\left(\mathscr{O}_{Y} / \mathscr{I}\right)$ and $\overline{y_{1}}$ (the scheme-theoretic image of $y_{1}$ in $Y$ ) are contained in $Z$. But $\overline{y_{1}}$ has one associated point, and $\operatorname{Spec}_{X}\left(\mathscr{O}_{Y} / \mathscr{I}\right)$ has one fewer associated point than $Y$, so we are done by induction on the number of associated points.

Proposition 3.7. Let $K$ be a finite extension of $\mathbb{Q}_{p}$ with valuation ring $R$, uniformizer $\pi$, and residue field $k$. Let $X$ be a smooth, finite-type $R$-scheme with geometrically connected fibers. Let $Y$ and $Z$ be closed subschemes of $X$; let $f: X \rightarrow X$ be an étale $R$-endomorphism. Then the set of $m \geqslant 0$ such that $f^{m}(Y) \subset Z$ is a one-sided semilinear set, whose period depends only on the associated points of $Y$.

Furthermore, if $f: X \rightarrow X$ is an automorphism, then the set of $m$ such that $f^{m}(Y) \subset Z$ is a two-sided semilinear set, with length bounded in the same way.

Proof. The idea of the proof is to replace $f$ with an iterate so that it fixes a point in the closure of every associated point of $Y$. Then Lemma 3.6 reduces the claim to a local statement, which we may prove via Lemma 3.3. We now give the details.

Let $r$ be such that for each associated point $y$ of $Y$, the closure $\bar{y}$ contains a $\mathbb{F}_{q^{r}}$-point. The map $X\left(\mathbb{F}_{q^{r}}\right) \rightarrow X\left(\mathbb{F}_{q^{r}}\right)$ induced by $f$ has eventual image

$$
I=\bigcap_{n} f^{n}\left(X\left(\mathbb{F}_{q^{r}}\right)\right)
$$

which is permuted by $f$; hence $f^{N}$ for some $N \gg 0$ fixes the eventual image $I$. Let $R^{\prime}$ be an unramified extension of $R$ such that each point of $I$ is the image of some $R^{\prime}$ point of $X$; replace $R$ with $R^{\prime}$. Let $g=f^{N}$; it suffices to show that the set of $m$ such that $g^{m}\left(f^{i}(Y)\right) \subset Z$ for $i=0, \ldots, N-1$ is a semilinear set. 


\section{J. LESIEUTRE AND D. LitT}

Now, given $x \in I$ with ideal sheaf $\mathfrak{m}_{x}$, fix an isomorphism

$$
\widehat{\mathscr{O}_{X, x}}=\lim _{\longleftarrow} \mathscr{O}_{X} / \mathfrak{m}_{x}^{n} \simeq R\left[\left[x_{1}, \ldots, x_{n}\right]\right] .
$$

As $f$ is étale, $f^{N}$ induces an isomorphism

$$
\widehat{\mathscr{O}_{X, x}} \rightarrow \widehat{\mathscr{O}_{X, x}}
$$

for each $x \in I$. By Lemma 3.4, the hypotheses of Lemma 3.3 are satisfied for this map. Hence, the set of $m$ such that $g^{m}\left(f^{i}(Y)\right) \cap \operatorname{Spf}\left(\widehat{\mathscr{O}_{X, x}}\right) \subset Z \cap \operatorname{Spf}\left(\widehat{\mathscr{O}_{X, x}}\right)$ is semilinear for each $x \in I$. By Lemma 3.6, this implies the global result we desired.

Remark. In fact, the period of the semilinear sets in the statement above does not depend on the supports of the associated points of $Y$ and $Z$, but rather on $X$ and the minimal $k$ such that

$$
\bar{\xi}\left(\mathbb{F}_{q^{k}}\right) \neq \emptyset
$$

for all associated points $\xi$ of $Y$ and $Z$. Here $\bar{\xi}$ denotes the Zariski closure of $\xi$. This yields the following corollary, which will prove useful later on.

Corollary 3.8 (Uniform Skolem-Mahler-Lech theorem). Let $f: \mathbb{Z}^{r} \rightarrow \mathbb{Z}^{r}$ be a linear automorphism, and let $x, y \in \mathbb{Z}^{r}$ be two elements. Then

$$
A(x, y):=\left\{n: f^{n}(x)=y\right\}
$$

is two-sided semilinear with period bounded only in terms of $r$.

Proof. This is immediate from the remark above, after extending scalars to $\mathbb{Z}_{3}$. (It can also be checked by easy linear algebra.)

The results above imply Theorems 1.2 and 1.3. The final step in the argument is to reduce the theorems for varieties over arbitrary fields of characteristic zero to varieties over extensions of the $p$-adics. To do this, it is necessary to produce a single finite-type integral $\mathbb{Z}$-algebra $R$ over which the infinitely many closed subschemes $Y_{j}$ are all defined. We now turn to the proof of Theorem 1.3, which includes Theorem 1.2 as a case.

Proof of Theorem 1.3. The statement is insensitive to base change along a field extension $k \subset k^{\prime}$, so we may, without loss of generality, assume that $k$ is uncountable. form

We first prove claim (i) of Theorem 1.3. Since the schemes $Y_{j}$ are all defined by ideals of the

$$
\mathscr{I}_{Y_{j}}=\sum_{i} \mathscr{I}_{Y_{i}^{0}}^{n_{i}}
$$

for some finite set of closed subschemes $Y_{i}^{\circ}$, there exist a finite-type integral $\mathbb{Z}$-algebra $R, R$ schemes $X^{\prime}, Y_{i}^{\prime}, Z^{\prime}$, an étale endomorphism $f^{\prime}$ of $X^{\prime}$, and a flat map $\iota: \operatorname{Spec}(k) \rightarrow \operatorname{Spec}(R) \operatorname{such}$ that $X, Y_{i}, Z, f$ are obtained by base change along $\iota$. Without loss of generality, we may assume that $X^{\prime}$ is smooth with geometrically connected fibers.

The claim of the theorem is then a consequence of Proposition 3.7, as follows. For each $j$, let $Y_{j}^{\prime \prime}$ be the subscheme of $X^{\prime}$ defined by the sheaf of algebras $\mathscr{O}_{Y_{j}^{\prime}} / \mathscr{I}$, where $\mathscr{I}$ consists of all functions whose support does not contain the image of $x$ in $X^{\prime}$. (This modification serves to eliminate the associated points whose closure does not contain $x$.) Then by hypothesis, the set of associated points of the $\left\{Y_{j}^{\prime \prime}\right\}$ is contained in the set $\{\xi \in \Xi: x \in \bar{\xi}\}$, and hence is finite. So the $\left\{Y_{j}^{\prime \prime}\right\}$ have finitely many associated points, and thus by Proposition 3.7 we conclude that the 


\section{Dynamical Mordell-Lang AND AUtomorphisms of BLOW-UpS}

set $\left\{n: \phi^{n}\left(Y_{j}^{\prime \prime}\right) \subset Z^{\prime}\right\}$ is semilinear of period independent of $j$. Now, base changing to $k$ and restricting to the local ring at $x$ completes the proof.

For claim (ii), we proceed as above; as before, there exist a finite-type integral $\mathbb{Z}$-algebra $R$, $R$-schemes $X^{\prime}, Y_{i}^{\prime}, Z^{\prime}$, an étale endomorphism $f^{\prime}$ of $X^{\prime}$, and a flat map $\iota: \operatorname{Spec}(k) \rightarrow \operatorname{Spec}(R)$ such that $X, Y_{i}, Z, f$ are obtained by base change along $\iota$. Without loss of generality, we may assume that $X^{\prime}$ is smooth with geometrically connected fibers. On the generic fiber of $X^{\prime}$, the $Y_{j}^{\prime}$ have only finitely many associated points. Hence replacing $Y_{j}^{\prime}$ with the subscheme of $X^{\prime}$ defined by $\mathscr{O}_{Y_{j}^{\prime}} / \mathscr{I}$, where $\mathscr{I}$ is the ideal sheaf consisting of functions whose support is on the special fiber of $X^{\prime}$, we may assume that the $Y_{j}^{\prime}$ have finitely many associated points, all of which are on the generic fiber. Note that $Y_{j}$ is still the base change of $Y_{j}^{\prime}$ along the map $R \rightarrow k$. Now the result follows again from Proposition 3.7.

In general, the set $\Xi$ of associated points of the closed subschemes defined by $\sum_{i=1}^{r} \mathscr{I}_{Y_{i}^{\circ}}^{n_{i}}$ need not be finite, as we saw in Example 2.7. In our applications of Theorem 1.3, we will only use part (i) of the theorem, which is generally easier to check. However, in some circumstances, the stronger hypothesis needed for part (ii) holds automatically.

Theorem 3.9. Let $X$ be a smooth variety, and let $\left\{Y_{1}^{\circ}, \ldots, Y_{r}^{\circ}\right\}$ be a finite set of closed subschemes of $X$. Let $Y_{j}$ be an infinite set of closed subschemes of the form

$$
\sum_{i=1}^{r} \mathscr{I}_{Y_{i}^{\circ}}^{n_{i}}
$$

for integers $n_{i} \geqslant 0$. If any of the following conditions hold, then the set of associated points of $Y_{j}$ is finite:

(i) Each $Y_{i}^{\circ}$ is a Cartier divisor, the $Y_{i}^{\circ}$ intersect transversely, and $Y_{j}$ is any collection of subschemes of the form $\sum_{i=1}^{r} \mathscr{I}_{Y_{i}^{\circ}}^{n_{i}}$.

(ii) We have $r=2$, and the $Y_{j}$ are defined by ideal sheaves $\mathscr{I}_{Y_{1}^{\circ}}+\mathscr{I}_{Y_{2}^{\circ}}^{n}$.

(iii) All but finitely many of the $Y_{j}$ are zero-dimensional.

Proof. In each case, it is sufficient to show that there are only finitely many associated points in any affine chart.

Case (i) follows from the observation that if $R$ is a regular ring and $f_{i}$ is a sequence of regular elements, then the set

$$
\bigcup_{n_{1}, \ldots, n_{r}} \operatorname{Ass}\left(R /\left(f_{1}^{n_{1}}, \ldots, f_{r}^{n_{r}}\right)\right)
$$

is finite.

For case (ii), we must show that if $R$ is a noetherian ring and $I$ and $J$ are two ideals in $R$, the set

$$
\bigcup_{n} \operatorname{Ass}\left(R /\left(I+J^{n}\right)\right)
$$

is finite. Observe that $R /\left(I+J^{n}\right) \cong(R / I) /\left(J^{n}(R / I)\right)$ as $R$-modules. But for any $R$-module $M$ and ideal $I$, the associated primes $\operatorname{Ass}\left(M / I^{n} M\right)$ stabilize for large $n$ [Bro79].

Case (iii) is immediate since the only possible associated points are the components of $Y_{j}$. 


\section{J. Lesieutre AND D. LitT}

\section{Local separation by blow-ups}

We now deduce some geometric consequences of the results of the previous section. Before beginning the proof of Theorem 1.4, we collect a few preliminary results. First, it will often be convenient to invoke the following.

TheOrem 4.1 ([Kol07, 3.4.1]). Suppose that $\phi: X \rightarrow X$ is an automorphism of a singular variety. There exists a birational map $\pi: Y \rightarrow X$ such that $Y$ is smooth and $\phi$ lifts to an automorphism of $Y$.

We now begin the proof of the local result on separation by blow-ups. For the remainder of this section, we work in the setting of Theorem 1.4. Let us fix some notation: we write $\mathscr{I}_{D}$ for the ideal sheaf on $X$ associated with the divisor $D$, and $\mathscr{I}_{V}$ for the ideal sheaf of $V$. We write $\mathscr{I}_{D, V}$ for the ideal in the local ring $\mathscr{O}_{X, V}$ determined by $\mathscr{I}_{D}$, and $\mathfrak{m}_{V}$ for the maximal ideal in $\mathscr{O}_{X, V}$. For simplicity, we write $\phi(\mathscr{I})$ for the ideal sheaf $\operatorname{im}\left(\left(\phi^{-1}\right)^{*} \mathscr{I} \rightarrow \mathscr{O}_{X}\right)$, so that if $W$ is a subscheme, we have $\phi\left(\mathscr{I}_{W}\right)=\mathscr{I}_{\phi(W)}$.

For each $k \geqslant 0$, let $D^{(k)}$ be the closed subscheme of $X$ defined by the ideal sheaf $\mathscr{I}_{D}+\mathscr{I}_{V}^{k+1}$. This is the $k$ th-order germ of $V$ along $D$. Then, consider the set

$$
A_{k}=\left\{n: \phi^{n}\left(D^{(k)}\right) \subseteq D \text { in } \operatorname{Spec}\left(\mathscr{O}_{X, V}\right)\right\} .
$$

By the first part of Theorem 1.3, applied with $x$ the generic point of $V$, there exists an $N$ such that each set $A_{k}$ is an $N$-periodic semilinear set. The hypothesis that only finitely many associated points of the $D^{(k)}$ contain $x$ clearly holds, since the only possible such points are the generic point of $D$ and $x$ itself.

To begin, we give some lemmas showing that after replacing $\phi$ by a suitable iterate, the sets $A_{k}$ take a particularly simple form. Note that when $\phi$ is replaced by an iterate $\phi^{n}$, the set $A_{k}$ is replaced by $\frac{1}{n}\left(A_{k} \cap n \mathbb{Z}\right)$.

The next lemma is the key application of Theorem 1.3: in the 2-dimensional setting, it implies that there is a uniform bound on the order of tangency between $C$ and the curves $\phi^{n}(C)$. The crucial observation is that because of the uniform bound $N$ on the periods of the arithmetic progressions in $A_{k}$, if any $\phi^{k}(D)$ is tangent to $D$ to order $j$, this tangency must occur for some $0<k \leqslant N$.

Lemma 4.2. There exists some $k$ for which $A_{k}$ is a finite set.

Proof. Let $Y_{1}^{\circ}=D$ and $Y_{2}^{\circ}=V$, and consider the collection of subschemes $D^{(k)}$ defined by the ideal sheaves $\mathscr{I}_{D}+\mathscr{I}_{V}^{k+1}$. It follows from Theorem 1.3(i) applied to these ideal sheaves, with $x$ the generic point of $V$, that if $A_{k}$ is an infinite set, then it contains an infinite arithmetic progression $P$ with step size dividing $N$ by Theorem 1.3. In particular, there must exist some $i$ with $1 \leqslant i \leqslant N$ for which $\phi^{i}\left(D^{(k)}\right) \subseteq D$ in $\mathscr{O}_{X, V}$.

Since $D$ is not periodic under $\phi$, the divisors $D$ and $\phi^{i}(D)$ are distinct for any nonzero $i$, and so for any $i$, there is a maximal $j$ for which $D^{(j)} \subset D \cap \phi^{i}(D)$ in $\mathscr{O}_{X, V}$. The claim of the lemma then holds with

$$
k=\max _{1 \leqslant i \leqslant N}\left(\max \left\{j: D^{(j)} \subset D \cap \phi^{i}(D)\right\}\right) .
$$

Lemma 4.3. Suppose that $\left\{A_{0}, \ldots, A_{n}\right\}$ is a finite collection of semilinear sets. Then there exists an integer $N$ such that $A_{i} \cap N \mathbb{Z}$ is either empty, $\{0\}$, or $N \mathbb{Z}$ for each $i$. 


\section{Dynamical Mordell-Lang AND AUtomorphisms of BLOW-UpS}

Proof. Let $k$ be an integer divisible by the period of every arithmetic progression appearing in any of the sets $A_{i}$. Each $A_{i}$ can then be written as the union of a finite number of residue classes $k \mathbb{Z}+r$ and a finite set. Then $A_{i} \cap k \mathbb{Z}$ is either all of $k \mathbb{Z}$ or a finite set for each $i$. Replacing $k$ by a sufficiently large multiple $N$, we may eliminate any nonzero element of $A_{i} \cap k \mathbb{Z}$.

Lemma 4.4. Suppose that

$$
\mathbb{Z}=A_{0} \supseteq \cdots \supseteq A_{k-1} \supseteq A_{k} \supseteq A_{k+1} \supseteq \cdots
$$

is a decreasing chain of semilinear sets such that $A_{k}$ is finite for sufficiently large $k$ and each $A_{k}$ contains 0 . Then there exist an $n>0$ and a $k$ such that $A_{i} \cap n \mathbb{Z}=n \mathbb{Z}$ for $i \leqslant k$ and $A_{i} \cap n \mathbb{Z}=\{0\}$ for $i>k$.

Proof. According to Lemma 4.2, there exists some $m$ such that $A_{m}$ is a finite set. By Lemma 4.3 applied to the sets $A_{0}, \ldots, A_{m}$, there exists an $N$ such that for $1 \leqslant i \leqslant m$, we have either $A_{i} \cap N \mathbb{Z}=\{0\}$ or $A_{i} \cap N \mathbb{Z}=N \mathbb{Z}$, with $A_{0} \cap N \mathbb{Z}=N \mathbb{Z}$ and $A_{m} \cap N \mathbb{Z}=\{0\}$. Since the sets are decreasing, the claim follows.

It follows that there exists an $n$ such that after replacing $\phi$ by the iterate $\phi^{n}$, there exists a $k$ with $A_{i}=\mathbb{Z}$ for $i \leqslant k$ and $A_{i}=\{0\}$ for $i>k$.

Remark. The geometric significance of Lemma 4.4 to the discussion in Section 2 is this: it might happen, for example, that $\phi^{n}(C)$ is tangent to $C$ at $x$ whenever $n$ is 0 or 1 modulo 3 , while $C$ is tangent to $\phi^{n}(C)$ to order 2 only for a finite set $n=3,6,7$. In this setting, we have $N=3$ and take $k_{0}=1$. After replacing $\phi$ by $\phi^{9}$, we arrange that

(1) $C$ is tangent to $\phi^{n}(C)$ for all $n$,

(2) $C$ is not tangent to $\phi^{n}(C)$ to order 2 for any $n$.

The first step in the proof is to show that $\mathscr{I}_{D, V}+\mathscr{I}_{\phi^{n}(D), V}$ stabilizes to an ideal independent of $n$; the means roughly that the multiplicity of intersection of $D$ and $\phi^{n}(D)$ along $V$ is independent of $n$.

We do this first in the case that $D$ is smooth at the generic point of $V$. This follows from calculations in the local ring at $V$.

Lemma 4.5. Let $\phi: X \rightarrow X$ be an automorphism of smooth variety over a field of characteristic zero and $V$ a codimension 2 subvariety with $\phi(V)=V$. Suppose that $D$ is an irreducible divisor containing $V$ that is smooth at the generic point of $V$ and not $\phi$-periodic. Then after replacing $\phi$ by an iterate, there exists a value of $k$ such that

(i) $\mathscr{I}_{D, V}+\mathscr{I}_{\phi^{n}(D), V}=\mathscr{I}_{D, V}+\mathfrak{m}_{V}^{k+1}$ in $\mathscr{O}_{X, V}$ for every nonzero value of $n$;

(ii) there exists an ideal sheaf $\mathscr{I}_{W}$ on $X$ with $\phi^{n}\left(\mathscr{I}_{W}\right)=\mathscr{I}_{W}$ for every $n$ and $\left.\mathscr{I}_{W}\right|_{\mathscr{O}_{X, V}}=$ $\mathscr{I}_{D, V}+\mathfrak{m}_{V}^{k+1}$.

Proof. By Lemma 4.4, we may replace $\phi$ by an iterate $\phi^{d}$ such that there exists a value of $k$ for which $A_{k}=\mathbb{Z}$ while $A_{k+1}=\{0\}$. This means that $\phi^{n}\left(D^{(k)}\right) \subseteq D$ in $\mathscr{O}_{X, V}$ for all $n$, while $\phi^{n}\left(D^{(k+1)}\right) \subseteq D$ in $\mathscr{O}_{X, V}$ only for $n=0$. At the level of ideals in $\mathscr{O}_{X, V}$, this yields $\mathscr{I}_{D, V} \subseteq$ $\mathscr{I}_{\phi^{n}(D), V}+\mathfrak{m}_{V}^{k+1}$, while $\mathscr{I}_{D, V} \nsubseteq \mathscr{I}_{\phi^{n}(D), V}+\mathfrak{m}_{V}^{k+2}$. Replacing $n$ by $-n$, we have $\mathscr{I}_{D, V} \subseteq \mathscr{I}_{\phi^{-n}(D), V}+$ $\mathfrak{m}_{V}^{k+1}$. Applying $\phi^{n}$ to both sides yields $\mathscr{I}_{\phi^{n}(D), V} \subseteq \mathscr{I}_{D, V}+\mathfrak{m}_{V}^{k+1}$. Similarly, $\mathscr{I}_{\phi^{n}(D), V} \nsubseteq \mathscr{I}_{D, V}+$ $\mathfrak{m}_{V}^{k+2}$. It follows that

$$
\mathscr{I}_{D, V}+\mathfrak{m}_{V}^{k+1}=\mathscr{I}_{\phi^{n}(D), V}+\mathfrak{m}_{V}^{k+1} \text {. }
$$




\section{J. LesieutRe AND D. LitT}

Let $W \subset X$ be the scheme-theoretic image of

$$
\operatorname{Spec} \mathscr{O}_{X, V} /\left(\mathscr{I}_{D, V}+\mathfrak{m}_{V}^{k+1}\right) \rightarrow \operatorname{Spec} \mathscr{O}_{X, V} \rightarrow X
$$

defined by an ideal sheaf $\mathscr{I}_{W}$. It must be that $\phi^{n}(W)$ is the scheme-theoretic closure of

$$
\begin{aligned}
\phi^{n}\left(\operatorname{Spec} \mathscr{O}_{X, V} /\left(\mathscr{I}_{D, V}+\mathfrak{m}_{V}^{k+1}\right)\right) & =\operatorname{Spec} \mathscr{O}_{X, V} /\left(\mathscr{I}_{\phi^{n}(D), V}+\phi^{n}\left(\mathfrak{m}_{V}^{k+1}\right)\right) \\
& =\operatorname{Spec} \mathscr{O}_{X, V} /\left(\mathscr{I}_{\phi^{n}(D), V}+\mathfrak{m}_{V}^{k+1}\right)=\operatorname{Spec} \mathscr{O}_{X, V} /\left(\mathscr{I}_{D}+\mathfrak{m}_{V}^{k+1}\right) .
\end{aligned}
$$

This shows that $W$ is itself $\phi$-invariant. Note that $\mathscr{I}_{W, V}=\mathscr{I}_{D, V}+\mathfrak{m}_{V}^{k+1}$.

Since $X$ is smooth, $\mathscr{O}_{X, V}$ is a 2 -dimensional regular local ring. The ideal $\mathscr{I}_{D, V} \subset \mathscr{O}_{X, V}$ is principal, and since $D$ is smooth at the generic point of $V$, the ideal $\mathscr{I}_{D, V}$ is generated by an element not contained in $\mathfrak{m}_{V}^{2}$. Then $\mathscr{O}_{X, V} / \mathscr{I}_{D, V}$ is a 1 -dimensional regular local ring, hence a discrete valuation ring. The ideals in $\mathscr{O}_{X, V}$ containing $\mathscr{I}_{D, V}$ correspond to ideals in $\mathscr{O}_{X, V} / \mathscr{I}_{D, V}$, which are of the form $\mathfrak{m}_{V}^{r}$. It follows that every ideal in $\mathscr{O}_{X, V}$ containing $\mathscr{I}_{D, V}$ is of the form $\mathscr{I}_{D, V}+\mathfrak{m}_{V}^{r}$ for some $r$.

We claim next that

$$
\mathscr{I}_{D, V}+\mathscr{I}_{\phi^{n}(D), V}=\mathscr{I}_{D, V}+\mathfrak{m}_{V}^{k+1}
$$

for every nonzero value of $n$. On the one hand, we have

$$
\mathscr{I}_{D, V}+\mathscr{I}_{\phi^{n}(D), V} \subseteq \mathscr{I}_{D, V}+\mathscr{I}_{\phi^{n}(D), V}+\mathfrak{m}_{V}^{k+1}=\mathscr{I}_{D, V}+\mathfrak{m}_{V}^{k+1} .
$$

To see that $\mathscr{I}_{D, V}+\mathscr{I}_{\phi^{n}(D), V}=\mathscr{I}_{D, V}+\mathfrak{m}_{V}^{k+1}$, it suffices to note that $\mathscr{I}_{D, V}+\mathscr{I}_{\phi^{n}(D), V} \nsubseteq$ $\mathscr{I}_{D, V}+\mathfrak{m}_{V}^{k+2}$ by the choice of $k$.

We now prove a special case of Theorem 1.4; we will later reduce the theorem to this case.

Theorem 4.6 (Local separation, smooth case). Let $X$ be a smooth variety over $k$ and $\phi: X \rightarrow X$ an automorphism. Suppose that $D \subset X$ is an irreducible divisor containing a codimension 2 irreducible closed subset $V$ with $\phi(V)=V$, that $D$ is not $\phi$-periodic, and that $D$ is smooth at the generic point of $V$. Then there exists a birational morphism $\pi: Y \rightarrow X$ such that

(i) $Y$ is smooth;

(ii) some iterate of $\phi$ lifts to an automorphism $\psi: Y \rightarrow Y$;

(iii) $\pi\left(\psi^{m}(\tilde{D}) \cap \psi^{n}(\tilde{D})\right)$ does not contain $V$ for any $m \neq n$.

Proof. Let $\pi_{0}: Y_{0} \rightarrow X$ be the blow-up of $X$ along the closed subscheme $W$ obtained from Lemma 4.5. We claim that

(1) an iterate of $\phi$ lifts to an automorphism $\psi_{0}: Y_{0} \rightarrow Y_{0}$;

(2) $\pi_{0}\left(\phi^{m}(\tilde{D}) \cap \phi^{n}(\tilde{D})\right)$ does not contain $V$ for $m \neq n$.

The first claim is immediate since the center $W$ is invariant under some iterate $\phi^{r}$ of $\phi$, by construction. Write $\psi_{0}: Y_{0} \rightarrow Y_{0}$ for this lift of $\phi^{r}$.

By part (ii) of Lemma 4.5 , we have $\mathscr{I}_{D, V}+\mathscr{I}_{\phi^{n}(D), V}=\mathscr{I}_{D, V}+\mathfrak{m}_{V}^{k+1}$ in $\mathscr{O}_{X, V}$ for every nonzero value of $n$. Since this equality holds at the generic point of $V$, for every $n$ there exists an open set $U_{n} \subset X$ with $\left.\left(\mathscr{I}_{D}+\mathscr{I}_{\phi^{n}(D)}\right)\right|_{U_{n}}=\left.\mathscr{I}_{W}\right|_{U_{n}}$. It then follows from [Har77, Exercise 7.12] that the strict transforms of $D$ and $\phi^{n}(D)$ do not meet above $U_{n}$, so that $\pi_{0}\left(D \cap \psi_{0}^{n}(D)\right)$ is disjoint from $U_{n}$. This shows that the necessary disjointness holds for $m=0$. In general, we obtain

$$
\pi_{0}\left(\psi_{0}^{m}(\tilde{D}) \cap \psi_{0}^{n}(\tilde{D})\right)=\pi_{0}\left(\psi_{0}^{m}(\tilde{D}) \cap \psi_{0}^{n-m}(\tilde{D})\right)=\phi^{n-m}\left(\pi_{0}\left(\tilde{D} \cap \psi_{0}^{n-m}(\tilde{D})\right)\right) .
$$




\section{Dynamical Mordell-Lang AND AUtomorphisms of BLOW-UpS}

This set is consequently disjoint from $\phi^{n-m}\left(U_{n}\right)$, an open subset of $V$, as required. Hence, parts (ii) and (iii) of Theorem 4.6 are satisfied by the model $\pi_{0}: Y_{0} \rightarrow X$. However, the variety $Y_{0}$ may not itself be smooth. Applying Theorem 4.1 to $\psi_{0}: Y \rightarrow Y$, we obtain a smooth model $\pi: Y \rightarrow X$ on which all three conditions are satisfied, completing the proof.

Remark. Some additional care is needed to prove the local separation result (Theorem 1.4) in the case that $D$ is not smooth at the generic point of $V$, since the proof of Theorem 4.6 required that $\mathscr{I}_{D, V}$ is not contained in $\mathfrak{m}_{V}^{2} \subset \mathscr{O}_{X, V}$. We now show that it is possible to reduce to the case that $\tilde{D}$ is smooth at the generic point of $V$.

To see the difficulty, suppose that $X$ is a surface and $V$ is a point, as in Section 2. It might be that $D \subset X$ is a curve with a node at $V$. It is possible that one of the local branches of $D$ has tangent direction fixed by $\phi$, while the other does not; this is illustrated in Figure 2.

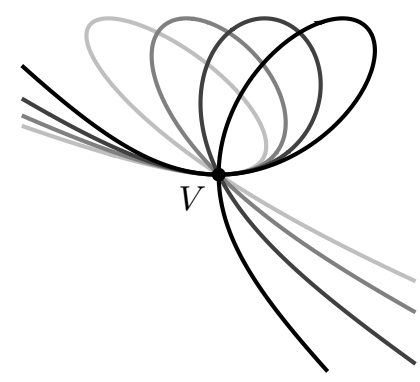

Figure 2. Orbit of a curve with a node at the fixed point

The automorphism lifts to the blow-up of $X$ at the node of $D$. One point of intersection of $\tilde{D}$ with the exceptional divisor has infinite orbit. The other intersection $x$ is a fixed point of the automorphism. However, the curve is smooth at $x$, and Theorem 4.6 can be applied to guarantee the existence of a model on which the iterates are separated.

Lemma 4.7. Suppose that $\phi: X \rightarrow X$ is an automorphism of a smooth variety over $k$ and that $V \subset X$ is an irreducible codimension 2 subvariety with $\phi(V)=V$. Suppose that $D \subset X$ is an irreducible divisor that contains $V$ and is not $\phi$-periodic. Then there exists a birational map $\pi: Y \rightarrow X$ such that

(i) $Y$ is smooth;

(ii) some iterate of $\phi$ lifts to an automorphism $\psi: Y \rightarrow Y$;

(iii) for every value of $n$, the codimension 2 part of $D \cap \phi^{n}(D) \cap \pi^{-1}(V)=\bigcup_{i} V_{i}$ is a union of finitely many $\psi$-invariant codimension 2 subvarieties $V_{i}$ of $Y$, and $D$ is smooth at the generic point of $V_{i}$ for each $i$.

Proof. We will construct $Y$ via a sequence of blow-ups $\pi_{k}^{\prime}: X_{k} \rightarrow X_{k-1}$ such that $\phi$ induces automorphisms $\psi_{k}: X_{k} \rightarrow X_{k}$. Write $\pi_{k}: X_{k} \rightarrow X$ for the composition of these blow-ups, and write $D_{k}$ for the strict transform of $D$ on $X_{k}$.

The argument is by induction on the maximal multiplicity of $D$ along a fixed component of $\psi_{k}$ lying above $V$. More precisely, let $m\left(X_{k}\right)$ be the number

$$
m\left(X_{k}\right)=\max _{\substack{V_{i} \subset \pi_{k}^{-1}(V) \\ \operatorname{codim}_{X} V_{i}=2 \\ \psi_{k}^{n}\left(V_{i}\right)=V_{i}}} \operatorname{mult}_{V_{i}}\left(D_{k}\right),
$$

the maximum multiplicity of $D$ along a $\psi_{k}$-periodic codimension 2 subset lying above $V$. Here 


\section{J. Lesieutre AND D. LitT}

" $\psi_{k}^{n}\left(V_{i}\right)=V_{i}$ " in the subscript means that this equality holds for some nonzero $n$, so that $V_{i}$ is $\psi_{k}$-periodic. Observe that the set of codimension 2 subvarieties $V_{i} \subset \pi_{k}^{-1}(V)$ with mult $_{V_{i}}\left(D_{k}\right) \geqslant 0$ is finite, so this maximum is taken over a finite set. Note too that replacing $\phi$ with an iterate does not change $m\left(X_{k}\right)$, and so we do this freely in what follows. Let

$$
n\left(X_{k}\right)=\#\left\{V_{i} \subset \pi^{-1}(V): \operatorname{codim}_{X_{k}} V_{i}=2, \psi_{k}^{n}\left(V_{i}\right)=V_{i}, \text { and } \operatorname{mult}_{V_{i}}\left(D_{k}\right)=m\left(X_{k}\right)\right\}
$$

be the number of components achieving this multiplicity.

The proof is by induction on $\left(n\left(X_{k}\right), m\left(X_{k}\right)\right)$, ordered lexicographically: we show that after a sequence of blow-ups, either the maximal multiplicity decreases, or the number of $V_{i}$ achieving this multiplicity decreases.

Suppose $m\left(X_{k}\right) \geqslant 2$, and fix a component $V_{k}^{0} \subset \pi_{k}^{-1}(V)$ which is $\psi_{k}$-periodic and has mult $_{V_{k}^{0}}\left(D_{k}\right)=m\left(X_{k}\right)$. Let $\pi_{k+1}^{\prime 0}: X_{k+1}^{0} \rightarrow X_{k}$ be the blow-up along $V_{k}$. Since $V_{k}$ is $\psi_{k}$-invariant, $\psi_{k}$ lifts to an automorphism $\psi_{k+1}^{0}: X_{k+1}^{0} \rightarrow X_{k+1}^{0}$. The variety $X_{k+1}^{0}$ might be singular, but taking a functorial resolution $\pi_{k}^{\prime}: X_{k+1} \rightarrow X_{k}$, we may assume that $X_{k+1}$ is smooth and that $\psi_{k}$ lifts to $\psi_{k+1}: X_{k+1} \rightarrow X_{k+1}$.

Let $W_{1}, \ldots, W_{r}$ be the codimension 2 components of $D_{k+1} \cap\left(\pi_{k+1}^{\prime}\right)^{-1}\left(V_{k}^{0}\right)$. Each of the sets

$$
A_{\psi_{k+1}}\left(W_{i}, W_{j}\right):=\left\{z \in \mathbb{Z}: \psi_{k+1}^{z}\left(W_{i}\right) \subset W_{j}\right\}
$$

is semilinear. By Lemma 4.3, after replacing $\psi_{k+1}$ by an iterate, we may assume that $A_{\psi_{k}}\left(W_{i}, W_{i}\right)$ is either all of $\mathbb{Z}$ or empty, and that $A_{\phi}\left(W_{i}, W_{j}\right)$ is empty for $i \neq j$.

Then, suppose that $D_{k+1} \cap \psi_{k+1}^{n}(D) \cap \pi_{k+1}^{-1}\left(V_{k}^{0}\right)$ has a component with codimension 2 in $X$. It must be that $\phi^{n}\left(W_{i}\right)=W_{j}$ for some $i$ and $j$. But by the above, this is possible only if $i=j$ and $W_{i}$ is $\phi$-invariant.

We have mult $_{W_{i}}\left(D_{k+1}\right) \leqslant \operatorname{mult}_{V_{k}^{0}}\left(D_{k}\right)$ for any $i$, so $m\left(X_{k+1}\right) \leqslant m\left(X_{k}\right)$. However, it might happen that equality holds for some component $W_{i}$, so that $m\left(X_{k+1}\right)=m\left(X_{k}\right)$ and $n\left(X_{k+1}\right)=$ $n\left(X_{k}\right)$. In this case, we repeat the preceding blow-up procedure, taking $V_{k+1}^{0}=W_{i}$ for the center of the blow-up. If $m\left(X_{k}\right)$ and $n\left(X_{k}\right)$ do not decrease, we continue the process, obtaining a sequence of models $\cdots \rightarrow X_{k+1} \rightarrow X_{k}$ such that

(1) some iterate of $\phi$ lifts to an automorphism of $X_{k+\ell}$ for all $\ell \geqslant 0$;

(2) $m\left(X_{k+1}\right)=m\left(X_{k}\right)$ and $n\left(X_{k+1}\right)=n\left(X_{k}\right)$.

We claim that such a sequence of blow-ups must be finite: this follows from the standard local calculation showing that embedded resolution of singularities of curves on a surface can be achieved by repeatedly blowing up the singular points. Indeed, we may reduce the statement to the 2-dimensional case as follows. Fix a surface $S \subset X$ such that $S$ is transverse to $V$. Write $S_{k} \subset X_{k}$ for the strict transform of $S$ on $X_{k}$. Then for every $\ell \geqslant k$, we have

$$
\operatorname{mult}_{V_{\ell}^{0} \cap S_{\ell}}\left(\left.D_{\ell}\right|_{S_{\ell}}\right)=\text { mult }_{V_{\ell}^{0}} D_{\ell} \geqslant 2 \text {. }
$$

Since $S_{k+1}$ is obtained by blowing up $S_{k}$ at the points $V_{k}^{0} \cap S_{k}$, this sequence of blow-ups must be finite by [Kol07, Lemma 1.68]. This shows that the multiplicity eventually decreases, and we have either $n\left(X_{\ell}\right)<n\left(X_{k}\right)$ for some $\ell>k$ (if some other component $V_{k}^{\prime}$ has the same multiplicity as $V_{k}$, that is, if $n\left(X_{k}\right) \geqslant 2$ ) or $m\left(X_{\ell}\right)<m\left(X_{\ell}\right)$ (if $n\left(X_{k}\right)=1$ ). As a result, we eventually reach a model $Y=X_{\ell}$ on which $m\left(X_{\ell}\right)=1$.

Each model in our sequence has the property that if $D_{k} \cap \psi_{k}^{n}(D)$ has a component contained in $\pi_{k}^{-1}(V)$, this component is $\phi$-invariant. That $m\left(X_{\ell}\right)=1$ means that $D$ is smooth at the generic point of each such component, and so condition (iii) holds as required. 


\section{Dynamical Mordell-Lang AND AUtomorphisms of BlOW-UPS}

We finally prove Theorem 1.4. The statement is identical to that of Theorem 4.6, except that it is no longer assumed that $D$ is smooth at the generic point of $V$.

Proof of Theorem 1.4. We first apply Lemma 4.7 to obtain a birational map $\pi_{0}: Y_{0} \rightarrow X$ satisfying conditions (i)-(iii) of the lemma, so that $\phi$ lifts to an automorphism $\psi_{0}: Y_{0} \rightarrow Y_{0}$. Let $V_{1}, \ldots, V_{r}$ be the finitely many codimension $2 \psi_{0}$-invariant subsets of $\pi^{-1}(V)$. According to condition (iii), $\tilde{D}$ is smooth at the generic point of each $V_{i}$. Applying Theorem 4.6 to each $V_{i}$, we then obtain the desired blow-up $\pi: Y \rightarrow X$

To see that condition (iv) holds on $Y$, note that by condition (iii) of Lemma 4.7 on $Y_{0}$ we have $\psi_{0}^{m}(\tilde{D}) \cap \psi_{0}^{n}(\tilde{D}) \cap \pi^{-1}(V)$ equal to the union of the $V_{i}$ : these intersections have no components other than the $\psi_{k}$-invariant sets. By point (iii) of Theorem 4.6, we find that $\pi\left(\psi^{m}(\tilde{D}) \cap \psi^{n}(\tilde{D})\right)$ does not contain $V$.

\section{Global separation by blow-ups}

The following result on varieties containing infinite sets of disjoint divisors will play a role in the proof of Theorem 1.5.

Proposition 5.1 ([BPS16, Theorem 1.1]). Suppose that $X$ is a normal projective variety defined over an algebraically closed field and that $\left\{D_{i}\right\}$ is an infinite set of pairwise disjoint irreducible divisors on $X$. Then there exist a smooth projective curve $C$ and a map $f: X \rightarrow C$ such that each $D_{i}$ is contained in a fiber of $f$.

We also need the following easy lemma.

Lemma 5.2. Suppose that $\pi: Y \rightarrow X$ is a birational morphism between smooth projective varieties and that $f: Y \rightarrow C$ is a nonconstant morphism to a smooth curve which does not descend to $X$. Then $C$ is isomorphic to $\mathbb{P}^{1}$.

Proof. Some positive-dimensional fiber of $\pi$ must dominate $C$, as $f$ does not descend to $X$. But such fibers are rationally connected, so $C$ is $\mathbb{P}^{1}$.

We now prove Theorem 1.5. The strategy is to repeatedly apply Theorem 1.4 to components of $D \cap \phi^{n}(D)$, eventually rendering the infinitely many divisors $\phi^{n}(D)$ pairwise disjoint.

Theorem 5.3. Let $X$ be a smooth variety over $k$ and $\phi: X \rightarrow X$ an automorphism. Suppose that $D \subset X$ is an irreducible divisor such that

$$
V(D, \phi)=\left\{d \in D: \phi^{n}(d) \in D \text { for some nonzero } n\right\}=\bigcup_{n \neq 0} D \cap \phi^{n}(D)
$$

is a proper Zariski-closed subset of $D$. Then there exists a projective birational morphism $\pi: Y \rightarrow X$ such that

(i) $Y$ is smooth;

(ii) some iterate of $\phi$ lifts to an automorphism $\psi: Y \rightarrow Y$;

(iii) the divisors $\psi^{n}(\tilde{D})$ are pairwise disjoint.

Proof. The proof is by induction on the number of components of $V(D, \phi)$. If $V(D, \phi)$ is empty, claims (i)-(iii) of the theorem are already satisfied. Observe that replacing $\phi$ by an iterate can serve only to shrink the set $V(D, \phi)$ and decrease the number of components, and so we freely replace $\phi$ by iterates in what follows. 


\section{J. LESIEUTRE AND D. LitT}

We now claim that there exists a sequence $n_{i}$ with $\left|n_{i}\right|$ unbounded, such that $D \cap \phi^{n_{i}}(D)$ is nonempty for each $i$. Indeed, suppose that there exists an $N$ for which $\phi^{n}(D) \cap D$ is empty for all $n$ with $|n|>N$. Replacing $\phi$ by the iterate $\phi^{N}$, we may assume that $\phi^{n}(D) \cap D$ is empty for all $n \neq 0$. But then $\phi^{m}(D) \cap \phi^{n}(D)=\phi^{m}\left(D \cap \phi^{n-m}(D)\right)$ is empty for any $m \neq n$. Then claims (i), (ii), and (iii) hold with $\pi$ the identity map.

Let $V_{0}, \ldots, V_{r}$ be the finitely many components of $\bigcup_{n \neq 0} D \cap \phi^{n}(D)$, so that each $V_{i}$ is a codimension 1 subvariety of $D$. Since the intersections $D \cap \phi^{n_{i}}(D)$ are nonempty, there must exist some irreducible component $V=V_{j}$ which is contained in $D \cap \phi^{n_{i}}(D)$ for infinitely many of the $n_{i}$.

Thus the set $A_{\phi^{-1}}(V, D)=\left\{n: \phi^{-n}(V) \subset D\right\}=\left\{n: V \subset \phi^{n}(D)\right\}$ is infinite. By Lemma 4.4, after replacing $\phi$ by an iterate, we may assume either that $\phi^{n}(V)$ is not contained in $D$ for any $n$, or that it is contained in $V$ for all $n$. In the former case, $V$ is no longer contained in $V(D, \phi)$, and we continue the induction. So we may then assume that $V \subset D \cap \phi^{n}(D)$ for all $n$. Applying $\phi^{-n}$, we see that $\phi^{-n}(V) \subset D \cap \phi^{-n}(D)$ for all $n$. Since $\phi^{-n}(V)$ has codimension 1 in $D$, it must coincide with some component $V_{i}$, and since there are only finitely many $V_{i}$, we must have $\phi^{-n}(V)=\phi^{-m}(V)$ for some distinct $m$ and $n$. But then $\phi^{n-m}(V)=V$, and replacing $\phi$ by the iterate $\phi^{n-m}$, we may assume that $\phi(V)=V$.

We now apply the local statement of Theorem 1.4 to $\phi: X \rightarrow X$ along the invariant subvariety $V$ and obtain a model $\pi: Y \rightarrow X$ on which $\phi$ lifts to an automorphism and for which $\pi\left(\psi^{m}(\tilde{D}) \cap \psi^{n}(\tilde{D})\right)$ does not contain $V$. Now, suppose that $W \subset V(\tilde{D}, \psi)$ is a codimension 2 subvariety of $Y$. Then $W \subset \tilde{D} \cap \psi^{n}(\tilde{D})$ for some $n$, and $\pi(W) \subset D \cap \phi^{n}(D)$. This shows that the components of $V(\tilde{D}, \psi)$ are a subset of those of $V(D, \phi)$. Since the component $V$ has been removed and $\pi$ is an isomorphism away from $V$, the cardinality of this set decreases. Applying this procedure inductively, we eventually obtain a model $\pi: Y \rightarrow X$ satisfying conditions (i)-(iii).

Corollary 5.4. Let $\phi: X \rightarrow X$ be as in Theorem 5.3, and let $\pi: Y \rightarrow X$ be the birational model produced by the theorem. Assume in addition that $X$ is projective.

Then there exist a curve $C$, a morphism $f: Y \rightarrow C$, and an automorphism $\tau: C \rightarrow C$ such that $f \circ \psi=\tau \circ f$. Moreover, the normal bundle of $D$ satisfies $H^{0}\left(D, N_{D / X}\right)>0$ and $D$ moves in a positive-dimensional family on $X$. If $V(D, \phi)$ is nonempty, the divisor $D$ is linearly equivalent to a $\phi$-periodic divisor.

Proof. Since the divisors $\psi^{n}(\tilde{D})$ are pairwise disjoint, it follows from Proposition 5.1 that there exists a map $f: Y \rightarrow C$ such that all of the divisors $\psi^{n}(\tilde{D})$ are contained in fibers of $f$. The map $f$ has only finitely many reducible fibers, and so there are infinitely many $m$ for which $\psi^{m}(\tilde{D})$ and $\psi^{m+1}(\tilde{D})$ are both irreducible fibers of $f$.

Now, we show that $\psi$ induces an automorphism of $C$. The map $Y \rightarrow C$ is flat by, for example, the miracle flatness theorem, and hence induces a map $C \rightarrow \operatorname{Hilb}(Y)$. Let $B \subset \operatorname{Hilb}(Y)$ be the image of this map; $C$ is the normalization of $B$. The automorphism $\psi$ induces an automorphism $\psi_{H}: \operatorname{Hilb}(Y) \rightarrow \operatorname{Hilb}(Y)$. Since $\psi_{H}\left(\left[\psi^{m}(D)\right]\right)=\left[\psi^{m+1}(D)\right]$, we see that $\psi_{H}(B)$ meets $B$ infinitely many times. Since $B$ is 1-dimensional, it must be preserved by $\psi_{H}$. Consequently, $\psi$ induces an automorphism of $B$, and hence $C$. We call this morphism $\tau: C \rightarrow C$; it satisfies $\phi \circ f=f \circ \tau$ by construction.

The divisor $\tilde{D}$ is a fiber of $f: Y \rightarrow C$ and so moves in a 1-parameter family inside $Y$. It follows that $D$ moves in a 1-parameter family inside $X$ and that $H^{0}(X, D)>0$, as claimed.

By Lemma 5.2, if $V(D, \phi)$ is nonempty, $C$ must be isomorphic to $\mathbb{P}^{1}$. It follows that the divisors $\phi^{n}(D)$ are all linearly equivalent; as any automorphism of $\mathbb{P}^{1}$ has a fixed point, this implies that $D$ is linearly equivalent to a periodic divisor. 


\section{Dynamical Mordell-Lang AND AUtomorphisms of BlOW-UPS}

In combination, Theorem 5.3 and Corollary 5.4, complete the proof of Theorem 1.5.

\section{Applications to automorphism groups}

In this section, we will explain how the results of the previous section can be applied in a geometric context to study automorphism groups of varieties constructed by sequences of blow-ups. Before beginning the proof, we collect some preliminary observations about varieties with multiple contractible divisors.

Lemma 6.1. Suppose that $X$ is a smooth projective variety of dimension $n$ and that $\pi_{i}: X \rightarrow Y_{i}$, for $i \in I$, are distinct morphisms realizing $X$ as the blow-up of a smooth variety $Y_{i}$ at a center of dimension less than or equal to $r$.

(i) If $2 r+3 \leqslant n$, then any two exceptional divisors $E_{i}$ and $E_{j}$ either coincide or are pairwise disjoint. Moreover, the set of exceptional divisors $E_{i}$ is finite.

(ii) If $r+3 \leqslant n$ and $\operatorname{Nef}\left(E_{0}\right)$ is a rational polyhedral cone, then there exists a finite set of irreducible divisors $W_{i} \subset E_{0}$ such that if $E_{j}$ is distinct from $E_{0}$, then $E_{0} \cap E_{j} \subseteq \bigcup_{i} W_{i}$ for every $j \neq 0$.

Proof. First, we prove part (i). Suppose that some $E_{i}$ and $E_{j}$ have nonempty intersection, and choose a point $x$ in the intersection. Let $F \cong \mathbb{P}^{n-r-1}$ be the fiber of $\pi_{i}$ containing $x$ and $F^{\prime} \cong \mathbb{P}^{n-r-1}$ be the fiber of $\pi_{j}$ containing $x$. Since $F \cap F^{\prime}$ is nonempty and $n \geqslant 2 r+3$, the intersection of $F$ and $F^{\prime}$ must contain a curve $\Gamma$. The map $\pi$ contracts $F$ and hence $\Gamma$ to a point. But it is impossible to contract a positive-dimensional subvariety of $F^{\prime}$ without contracting all of $F^{\prime}$, as $F^{\prime} \simeq \mathbb{P}^{n-r-1}$. Hence $E_{i}=E_{j}$.

Now, we prove the finiteness claim. Let $f_{i}$ be a line contained in a fiber of $E_{i}$, so that $E_{i} \cdot f_{i}=-1$. Since $E_{i}$ is disjoint from $E_{j}$ for $i \neq j$, we must have $E_{i} \cdot f_{j}=0$ if $i \neq j$. This implies that the classes of the $E_{i}$ are linearly independent in $N^{1}(X)$. Since $N^{1}(X)$ is finite-dimensional, there can be at most finitely many contractions.

Next, we prove part (ii). Let $x$ be any point of $E_{0} \cap E_{j}$, and let $F_{0}$ and $F_{j}$ be the fibers of the maps $\pi_{0}$ and $\pi_{j}$ passing through $X$. Observe that $F_{j} \cap E_{0}$ is contracted to a point by $\pi_{j}$ and has dimension $n-r-2$. Since $r+3 \leqslant n$, this intersection has positive dimension. It follows that the divisor $E_{0} \cap E_{j} \subset E_{0}$ is contracted by the map $\pi_{j}$.

Any map $\left.\pi_{i}\right|_{E_{0}}: E_{0} \rightarrow \pi_{i}\left(E_{0}\right)$ contracting a divisor determines a codimension 1 face of $\operatorname{Nef}\left(E_{0}\right)$, and two contractions with different fibers determine different faces of the cone. Since $\operatorname{Nef}\left(E_{0}\right)$ has only finitely many faces by hypothesis, only finitely many divisors $W_{i}$ appear among the exceptional divisors of contractions $\left.\pi_{i}\right|_{E_{0}}$. Hence, the support of the intersection $E \cap \phi^{n}(E)$ must be contained in the union $W=\bigcup_{i} W_{i}$ for every value of $n$.

We will later apply this lemma in the case that $X$ is a variety with an infinite-order automorphism $\phi: X \rightarrow X$ and the maps $\pi_{i}: X \rightarrow Y_{i}$ are of the form $\pi_{0} \circ \phi^{-n}$, where $\pi_{0}: X \rightarrow Y_{0}$ is some fixed contraction. We also note that condition (ii) holds automatically if $\pi: Y \rightarrow X$ is the blow-up of $X$ along any variety of Picard rank 1, since then $E_{0}$ has Picard rank 2.

Lemma 6.2. Suppose that $\pi: Y \rightarrow X$ is the blow-up along a smooth subvariety with exceptional divisor E. Let $\phi: Y \rightarrow Y$ be an automorphism with $\phi(E)=E$. Then the iterate $\phi^{\rho(E)}$ descends to an automorphism of $X$. 


\section{J. LESIEUTRE AND D. LitT}

Proof. This is a case of [Les15, Lemma 3.2]. Briefly, it suffices to show that some iterate of $\phi$ sends fibers of $E$ to fibers of $E$, and then $\phi$ descends to an automorphism of $X$. This in turn follows from the fact that $E$ is a $\mathbb{P}^{n}$-bundle, and a given variety $E$ has at most $\rho(E)$ different $\mathbb{P}^{n}$-bundle structures [Wiś91, Theorem 2.2].

Lemma 6.3. Suppose that $\phi: X \rightarrow X$ is an automorphism of a smooth projective variety. There is a constant $e$ depending only on $\rho(X)$ (and not on $\phi$ ) such that if $D$ is a divisor which is rigid in its cohomology class (in the sense that $D$ is the only effective divisor with class $[D]$ in $N^{1}(X)$ ) and $\phi^{n}(D)=D$ for some positive $n$, there exists an $0<n^{\prime} \leqslant e$ with $\phi^{n^{\prime}}(D)=D$.

Proof. Since $\phi^{*}$ and its inverse are both given by invertible integer matrices, their determinant is \pm 1 . Corollary 3.8 provides an integer $e$ depending only on $\operatorname{rank} N^{1}(X)$ such that $\left(\phi^{*}\right)^{n^{\prime}}([D])=$ $[D]$ for some $n^{\prime} \leqslant e$. Since $D$ is the only divisor with class $[D]$, it must be that $\phi^{n}(D)=D$.

Lemma 6.3 does not follow from the bounds on the periods in Theorem 1.2, since the bounds there depend on the field of definition of $\phi$. The strategy is instead to conclude the statement from the corresponding statement for the linear map $\phi^{*}: N^{1}(X) \rightarrow N^{1}(X)$.

Proof of Theorem 1.6. We denote the sequence of blow-ups used in constructing $Y$ as follows:

$$
Y=X_{n} \stackrel{\pi_{n-1}}{\longrightarrow} X_{n-1} \stackrel{\pi_{n-2}}{\longrightarrow} \cdots \stackrel{\pi_{1}}{\longrightarrow} X_{1} \stackrel{\pi_{0}}{\longrightarrow} X_{0}=X
$$

Let $E_{j} \subset X_{j}$ be the exceptional divisor of $X_{j} \rightarrow X_{j-1}$ and $\tilde{E}_{j} \subset X$ its strict transform. The argument proceeds in two steps. We first show that the statement holds if all of the divisors $\tilde{E}_{j}$ are periodic under $\phi$. Then, we show that this hypothesis - that the $\tilde{E}_{j}$ are periodic-must in fact hold.

First, assume that the $\tilde{E}_{j}$ are all periodic under $\phi$. As each $\tilde{E}_{j}$ is rigid, by Lemma 6.3 , there is a constant $e(X)$ such that the period of $\tilde{E}_{j}$ divides $e(X)$. We claim that the iterate $\phi^{N}$ descends to an automorphism of $X$, where

$$
N=e(X) \prod_{i=1}^{n} \rho\left(E_{n}\right) .
$$

It follows from Lemma 6.3 that there is a constant $n_{0}=n_{0}(X)$ such that $\phi^{r}\left(E_{j}\right)=E_{j}$ for some $r$ less than $n_{0}$. Hence, replacing $\phi$ by $\phi^{r}$, we may assume that each divisor $\tilde{E}_{j}$ is invariant under $\phi$. Now, since $E_{n} \subset X_{n}$ is invariant under $\phi: X_{n} \rightarrow X_{n}$, the iterate $\phi^{\rho\left(E_{n}\right)}$ descends to an automorphism $\phi_{n-1}: X_{n-1} \rightarrow X_{n-1}$ by Lemma 6.2 . Continuing in this manner, we conclude that $\phi^{N}$ descends to an automorphism of $X$, as claimed.

Suppose that some $\tilde{E}_{j}$ is not periodic, and let $j$ be the largest index for which $E_{j}$ has infinite orbit. By the argument above, $\phi^{N}$ descends to an automorphism $\phi: X_{j} \rightarrow X_{j}$. Replace $Y$ by $X_{j}$, and let $\pi: X_{j} \rightarrow X_{j-1}$ be the contraction of $E_{j}$, for which we now write $E$. Then the maps $\pi \circ \phi^{-n}: X_{j} \rightarrow X_{j-1}$ give an infinite set of contractions, all with distinct exceptional divisors $\phi^{n}(E)$. In the case $2 r+3 \leqslant n$, this gives an immediate contradiction by Lemma 6.1.

If, instead, $r+3 \leqslant n$ and $\operatorname{Nef}(E)$ is polyhedral, applying Lemma 6.1 as before, we conclude that $\phi^{n}(E) \cap E$ is a union of the finitely many components $W_{i} \subset E$, independent of $n$. This means that the set $V(E)=\bigcup_{n \neq 0} E \cap \phi^{n}(E)$ is either empty or contains only the components of $W$. By Theorem 1.5, it must be that $H^{0}\left(E, N_{E / X}\right)>0$. This gives a contradiction, since $E$ is the exceptional divisor of a blow-up.

We now obtain Corollaries 1.7 and 1.9 as a consequence. 


\section{Dynamical Mordell-Lang AND AUtomorphisms of BlOW-UPS}

Proof of Corollary 1.7. Since all the blow-ups are assumed to be along points and curves, they satisfy condition (ii) of Theorem 1.6: $n=4$ and $r \leqslant 1$. Moreover, each $E$ has Picard rank at most 2 and the polyhedrality of $\operatorname{Nef}(E)$ is immediate.

Proof of Corollary 1.9. Write $Y=\mathrm{Bl}_{Z}(X)$, and let $G \subset \underline{\text { Aut }}(X)$ be the closed subgroup scheme consisting of automorphisms of $X$ that preserve $Z$. By assumption, $\operatorname{Aut}(X)$ is of finite type, so the same is true for $G$. Let $M=\#\left|G / G^{0}\right|$, where $G^{0}$ is the connected component of the identity in $G$.

Note that $G$ is naturally a subgroup scheme of Aut $(Y)$. By Theorem 1.6, there exists an integer $N$ such that for any $\phi \in \operatorname{Aut}(Y)$, we have $\phi^{N} \overline{\in G}$; thus $\phi^{N M}$ is in the identity component of $\underline{\operatorname{Aut}}(Y)$. In particular, $\underline{\operatorname{Aut}}(Y) / \underline{\operatorname{Aut}}^{0}(Y)$ is a group of bounded exponent.

Thus by Lemma 6.4, the natural map

$$
\operatorname{Aut}(Y) \rightarrow \mathrm{GL}\left(H^{*}(Y, \mathbb{C})\right)
$$

has finite image. Let $\operatorname{Aut}_{\omega}(Y)$ be the kernel of this map. By a well-known result of Lieberman-Fujiki [Lie78, Proposition 2.2], the subgroup scheme $\operatorname{Aut}^{0}(Y)$ has finite index in $\operatorname{Aut}_{\omega}(Y)$, which we have just shown has finite index in $\operatorname{Aut}(Y)$. Hence $\operatorname{Aut}(Y)$ has finite component group, as desired.

Lemma 6.4. Let $H \subset \mathrm{GL}_{n}(k)$ be a group of bounded exponent, with $k$ a field of characteristic zero. Then $H$ is finite.

Proof. Let $\bar{H}$ be the Zariski closure of $H$ in $\mathrm{GL}_{n}$. Then $\bar{H}$ is a subgroup scheme of $\mathrm{GL}_{n}$ which is of bounded exponent in the sense that if $N$ is the exponent of $H$, the map

$$
\bar{H} \stackrel{x \mapsto x^{N}}{\longrightarrow} \bar{H}
$$

is the same as the constant map $x \mapsto 1$. But now consider the group

$$
T:=\operatorname{ker}\left(\bar{H}\left(k[\epsilon] / \epsilon^{2}\right) \rightarrow \bar{H}(k)\right) .
$$

This is a torsion-free group (indeed, a $k$-vector space) of exponent $N$; hence, it must equal zero. Thus $\bar{H}$ is zero-dimensional, hence finite.

\section{Bounds on intersection multiplicities}

The applications in the preceding sections have focused on intersections $D \cap \phi^{n}(D)$ of a divisor with its own iterates under an automorphism of a variety. In fact, many of the same arguments can be applied to intersections with smaller-dimensional varieties. In the case that the intersection is zero-dimensional, this is a result of Arnold.

Lemma 7.1. Let $\phi: X \rightarrow X$ be an automorphism of a smooth variety, and let $V \subset X$ be a subvariety with $\phi(V)=V$. Suppose that $Y$ and $Z$ are two irreducible closed subvarieties of $X$ containing $V$, with $\operatorname{codim}_{X}(Z)=1$ and $\operatorname{codim}_{X} Y+\operatorname{codim}_{X} Z=\operatorname{codim}_{X} V$. Suppose further that $Y$ is regular at the generic point of $V$.

Then there exists some $K$ such that $\mathscr{I}_{V}^{K} \subseteq \mathscr{I}_{Y}+\mathscr{I}_{\phi^{n}(Z)}$ in $\mathscr{O}_{X, V}$ is satisfied for all nonzero $n$ such that $\operatorname{codim}_{\eta_{V}} Y \cap \phi^{n}(Z)=\operatorname{codim}_{\eta_{V}} V$. (Here, $\eta_{V}$ is the generic point of $V$ and $\mathscr{O}_{X, V}$ is the local ring of $X$ at the generic point of $V$.)

Furthermore, the set of $n$ such that $\operatorname{codim}_{\eta_{V}} Y \cap \phi^{n}(Z) \neq \operatorname{codim}_{\eta_{V}} V$ is semilinear.

Proof. Let $V_{k}$ be the scheme defined by $\mathscr{I}_{V}^{k}+\mathscr{I}_{Y}$. By Theorem 1.3, the set $A_{k}:=\left\{n: \phi^{n}\left(V_{k}\right) \subset Z\right\}$ is semilinear of period $N$, independent of $k$. Let $A_{\infty}=\cap_{k} A_{k}$. By Lemma 3.2, the intersection $A_{\infty}$ is also semilinear of period $N$. 


\section{J. Lesieutre AND D. LitT}

As semilinear sets of period $N$ satisfy the descending chain condition, there exists a $K$ such that $A_{K}=A_{\infty}$. By the regularity of $Y$ at the general point of $V$ and the fact that $V$ is of codimension 1 in $Y$ (by the hypothesis on $Z$ ), the local ring $\mathscr{O}_{Y, V}$ is a discrete valuation ring. Hence, the localization of $Y \cap \phi^{n}(Z)$ to the generic point of $V$ is either $\operatorname{Spec}\left(\mathscr{O}_{Y, V}\right)$ or $\operatorname{Spec}\left(\mathscr{O}_{Y, V} / \mathfrak{m}_{V}^{r}\right)$ for some $r$.

The intersection $A_{\infty}$ is the set of $n$ such that the localization of $Y \cap \phi^{n}(Z)$ to the generic point of $V$ is $\operatorname{Spec}\left(\mathscr{O}_{Y, V}\right)$ or, equivalently, $\operatorname{codim}_{\eta_{V}} Y \cap \phi^{n}(Z) \neq \operatorname{codim}_{\eta_{V}} V$, proving the last claim of the lemma.

Likewise, $A_{k}$ is the set of $n$ such that the localization of $Y \cap \phi^{n}(Z)$ to the generic point of $V$ is contained in $\operatorname{Spec}\left(\mathscr{O}_{Y, V} / \mathfrak{m}_{V}^{k}\right)$, which is contained in $\operatorname{Spec}\left(\mathscr{O}_{X, V} / \mathfrak{m}_{V}^{k}\right)$. Hence $\mathscr{I}_{V}^{K} \subseteq \mathscr{I}_{Y}+\mathscr{I}_{\phi^{n}(Z)}$ in $\mathscr{O}_{X, V}$ for all nonzero $n$ such that $\operatorname{codim}_{\eta_{V}} Y \cap \phi^{n}(Z)=\operatorname{codim}_{\eta_{V}} V$, as desired.

TheOREM 7.2 (cf. [Arn93]). Let $\phi: X \rightarrow X$ be an automorphism of a smooth variety, and let $V \subset X$ be a subvariety with $\phi(V)=V$. Suppose that $Y$ and $Z$ are two Cohen-Macaulay subvarieties of $X$ containing $V$, with $Y$ regular at the generic point of $V$, $\operatorname{codim}(Z)=1$, and $\operatorname{codim}_{X} Y+\operatorname{codim}_{X} Z=\operatorname{codim}_{X} V$. Then

$$
\left\{\operatorname{mult}_{V}\left(Y \cap \phi^{n}(Z)\right)\right\}
$$

is uniformly bounded in $n$, among $n$ with $V$ a component of $Y \cap \phi^{n}(Z)$ (that is, those $n$ for which this intersection has the expected dimension along $V$ ).

Proof. For $n$ for which the intersection is dimensionally correct, the intersection multiplicity along $V$ is defined by

$$
\operatorname{mult}_{V}\left(Y, \phi^{n}(Z)\right)=\sum_{i \geqslant 0}(-1)^{i} \operatorname{len}_{\mathscr{O}_{X, V}} \operatorname{Tor}_{i}^{\mathscr{O}_{X, V}}\left(\mathscr{O}_{Y, V}, \mathscr{O}_{\phi^{n}(Z), V}\right)
$$

However, by the Cohen-Macaulay assumption on $Y$ and $Z$, all terms except that with $i=0$ vanish, so that

$$
\operatorname{mult}_{V}\left(Y, \phi^{n}(Z)\right)=\operatorname{len}_{\mathscr{O}_{X, V}} \mathscr{O}_{X, V} /\left(\mathscr{I}_{Y, V}+\mathscr{I}_{\phi^{n}(Z), V}\right) \text {. }
$$

Since by Lemma 7.1, there exists a $k$ such that $\mathfrak{m}_{V}^{k} \subset \mathscr{I}_{Y, V}+\mathscr{I}_{\phi^{n}(Z), V}$ for all nonzero $n$ such that $Y \cap Z$ has the expected dimension at $V$, we have

$$
\operatorname{mult}_{V}\left(Y, \phi^{n}(Z)\right)=\operatorname{len}_{\mathscr{O}_{X, V}} \mathscr{O}_{X, V} /\left(\mathscr{I}_{Y, V}+\mathscr{I}_{\phi^{n}(Z), V}\right) \leqslant \operatorname{len}_{\mathscr{O}_{X, V}} \mathscr{O}_{X, V} / \mathfrak{m}_{V}^{k}
$$

a bound independent of $n$. This completes the proof.

It may happen that there are some values of $n$ for which $Y \cap \phi^{n}(Z)$ has larger than expected dimension, so that $M_{n}$ does not compute the multiplicity. Lemma 7.1 shows that this set is semilinear. However, if $Z$ is no longer a divisor, we do not know how to prove an analog of Theorem 7.2 except in the case $\operatorname{dim}(V)=0$ (the case covered by Arnol'd). Furthermore, we do not know how to control the set of $n$ such that $Y \cap \phi^{n}(Z)$ has larger than expected dimension.

Question 7.3. Suppose codim $(Y)>1$. Is the set of $n$ for which $Y \cap \phi^{n}(Z)$ has larger than expected dimension along $V$ a semilinear set?

\section{ACKNOWLEDGEMENTS}

We are grateful to Serge Cantat, Roland Roeder, Paul Reschke, and Kevin Tucker for useful conversations, and to János Kollár and David Stapleton for comments. 


\section{DynAmical Mordell-LANG AND AUTOMORPhisms OF BLOW-UPS}

\section{REFERENCES}

Arn93 V.I. Arnol'd, Bounds for Milnor numbers of intersections in holomorphic dynamical systems, Topological Methods in Modern Mathematics (Stony Brook, NY, 1991) (Publish or Perish, Houston, TX, 1993), 379-390.

BC13 T. Bayraktar and S. Cantat, Constraints on automorphism groups of higher dimensional manifolds, J. Math. Anal. Appl. 405 (2013), no. 1, 209-213; doi:10.1016/j.jmaa.2013.03.048.

BGT10 J.P. Bell, D. Ghioca, and T. J. Tucker, The dynamical Mordell-Lang problem for étale maps, Amer. J. Math. 132 (2010), no. 6, 1655-1675; https://muse.jhu.edu/article/404144.

BGT15_, Applications of p-adic analysis for bounding periods for subvarieties under étale maps, Int. Math. Res. Not. 2015 (2015), no. 11, 3576-3597; doi:10.1093/imrn/rnu046.

BL15 J.P. Bell and J. C. Lagarias, A Skolem-Mahler-Lech theorem for iterated automorphisms of K-algebras, Canad. J. Math. 67 (2015), no. 2, 286-314; doi:10.4153/CJM-2013-048-3.

BPS16 F. A. Bogomolov, A. Pirutka, and A. M. Silberstein, Families of disjoint divisors on varieties, Eur. J. Math. 2 (2016), no. 4, 917-928; doi:10.1007/s40879-016-0109-1.

Bro79 M. Brodmann, Asymptotic stability of $\operatorname{Ass}\left(M / I^{n} M\right)$, Proc. Amer. Math. Soc. 74 (1979), no. 1, 16-18; doi:10.2307/2042097.

Har77 R. Hartshorne, Algebraic geometry, Grad. Texts in Math., vol. 52 (Springer-Verlag, New York Heidelberg, 1977); doi:10.1007/978-1-4757-3849-0.

Kol07 J. Kollár, Lectures on resolution of singularities, Ann. of Math. Stud., vol. 166 (Princeton Univ. Press, Princeton, NJ, 2007).

Les15 J. Lesieutre, Some constraints on positive entropy automorphisms of smooth threefolds, Ann. Sci. Éc. Norm. Supér., to appear, arXiv:1503.07834.

Lie78 D. I. Lieberman, Compactness of the Chow scheme: applications to automorphisms and deformations of Kähler manifolds, Fonctions de plusieurs variables complexes, III (Sém. François Norguet, 1975-1977), Lecture Notes in Math., vol. 670 (Springer, Berlin, 1978), 140-186; doi:10.1007/BFb0064399.

Poo14 B. Poonen, p-adic interpolation of iterates, Bull. Lond. Math. Soc. 46 (2014), no. 3, 525-527; doi:10.1112/blms/bdu010.

SS04 A.K. Singh and I. Swanson, Associated primes of local cohomology modules and of Frobenius powers, Int. Math. Res. Not. 2004 (2004), no. 33, 1703-1733; doi:10.1155/S1073792804133424.

Tru17 T. T. Truong, Automorphisms of blowups of threefolds being Fano or having Picard number 1, Ergodic Theory Dynam. Systems 37 (2017), no. 7, 2255-2275; doi:10.1017/etds.2016.4.

Wiś91 J. A. Wiśniewski, On contractions of extremal rays of Fano manifolds, J. reine angew. Math. 417 (1991), 141-157; doi:10.1515/crll.1991.417.141.

John Lesieutre jdl@uic.edu

University of Illinois at Chicago, Math, Stat \& CS, 851 S Morgan St., Chicago, IL 60607, USA

Daniel Litt dlitt@math.columbia.edu

Department of Mathematics, Office 716, Columbia University, 2990 Broadway, New York, NY 10027, USA 